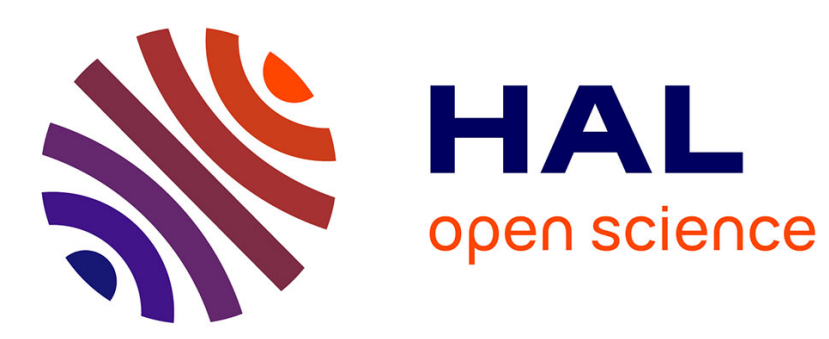

\title{
An asymptotic model for a thin, soft and imperfectly bonded elastic joint
}

Christian Licht, Gérard Michaille, Pongpol Juntharee

\section{To cite this version:}

Christian Licht, Gérard Michaille, Pongpol Juntharee. An asymptotic model for a thin, soft and imperfectly bonded elastic joint. Mathematical Methods in the Applied Sciences, 2016, 39 (5), pp.981997. 10.1002/mma.3535 . hal-02064947

\section{HAL Id: hal-02064947 https://hal.science/hal-02064947}

Submitted on 12 Mar 2019

HAL is a multi-disciplinary open access archive for the deposit and dissemination of scientific research documents, whether they are published or not. The documents may come from teaching and research institutions in France or abroad, or from public or private research centers.
L'archive ouverte pluridisciplinaire HAL, est destinée au dépôt et à la diffusion de documents scientifiques de niveau recherche, publiés ou non, émanant des établissements d'enseignement et de recherche français ou étrangers, des laboratoires publics ou privés. 


\title{
An asymptotic model for a thin, soft and imperfectly bonded elastic joint
}

\author{
Christian Licht $^{\mathrm{a}, \mathrm{b} \times \dagger}$, Gérard Michaillec and Pongpol Juntharee ${ }^{\mathrm{d}}$
}

\begin{abstract}
An asymptotic model for a thin, soft, linearly elastic joint imperfectly bonded to two linearly elastic bodies is derived by studying the variational convergence of the total mechanical energy when the thickness and the stiffness of the joint go to zero. The joint is replaced by a mechanical constraint between the adherents which correspond to the connection in series of the classical limit constraint induced by the soft joint and constraints between the joint and the adherents.
\end{abstract}

Keywords: asymptotic analysis; variational convergence; epigraphical sum; linearized elasticity; soft junctions; imperfect bonding

\section{Introduction}

Many studies have been devoted to asymptotic modeling of soft thin joints by considering the stiffness and thickness of the joints as small parameters. In the static case, the mathematically rigorous variational approach consists of determining the asymptotic behavior of minimizers of the total mechanical energy of the structure made of the adherents and elastic joint when the thickness and stiffness go to zero. This can be performed in the classical framework of displacement fields (see [1, 2] and the references therein, [3, 4]) or in an extended framework of measure fields [5], which may supply additional information at a lower scale. Anyway, the starting point is to assume that the joint and adherents are perfectly stuck together so that the asymptotic model consists of replacing the physical joint by an abstract mechanical constraint between the two adherents. This constraint keeps the memory of the joint, which disappears at the limit: its surface energy density $W$ strongly depends on the relative behavior of the parameters but can be written in a unified way with a mathematical structure similar to that of the bulk energy density of the genuine joint.

Here, under the small strains assumption, we assume that the joint and adherents are not perfectly stuck together so that the reversible mechanical constraint between them is not pure adhesion but is given by some smooth or nonsmooth convex surface energy densities $h^{1}$ and $h^{2}$. We will show that the asymptotic model consists of replacing the joint by a constraint, which is now the inf-convolution (or epigraphical sum) of the densities $h^{i}$ and the limit density $W$. This corresponds to the connection in series of the limit constraint induced by the joint and the constraints between the joint and adherents.

For the sake of clarity, in Section 2, we introduce a simplified situation where the joint connects an elastic body to a rigid support: the joint is clamped on the rigid body while there is a mechanical constraint between it and the elastic body. This situation was considered in [6] in the particular case of a linearly elastic adhesive and adherent and of bilateral contact with Tresca-like sliding between them. Their asymptotic analysis uses rescaling of the coordinates through the joint and a mixed formulation (with two fields: displacements and stress) in terms of variational inequality, so that the various limit mechanical constraints are only specified via graphs (relationships between the stress vector and the displacement). On the contrary, as it is obvious from the mechanical standpoint to guess the structure of the energy density of the limit constraint (inf-convolution is the mathematical translation of connection in series), we prefer to deal directly with the total energy functional and study its variational convergence; this is easily performed by adapting the arguments of [1] to this framework, which is indeed simpler because it is convex. Then, in Section 3, we propose our limit model which takes into account the asymptotic behavior of the displacement inside the adherent and inside the adhesive. The proofs of our statements are given in Section 4. Section 5 is devoted to a variant that is important in Tribology (the "third body" concept), where the thin adhesive layer

\footnotetext{
a Laboratoire de Mécanique et Génie Civil, UMR 5508 CNRS - UM2, Université Montpellier 2, c.c. 048, Place Eugène Bataillon, 34095 Montpellier Cedex 5, France

${ }^{b}$ Department of Mathematics, Mahidol University, Rama VI road Bangkok 10400, Thailand

C I3M, UMR CNRS 5149-UM2, Université Montpellier 2, c.c. 51, Place Eugène Bataillon, 34095 Montpellier Cedex 5, France

${ }^{d}$ Department of Mathematics, King Mongkut's University of Technology North Bangkok, 1518 Pibulsongkram road, Bangsue, Bangkok 10800, Thailand

* Correspondence to: Christian Licht, Laboratoire de Mécanique et Génie Civil, UMR 5508 CNRS - UM2, Université Montpellier 2, c.c. 048 , Place Eugène Bataillon, 34095 Montpellier Cedex 5, France.

†E-mail:clicht@univ-montp2.fr
} 
contains a far thinner and softer layer in the vicinity of the elastic adherent. In Section 6, we focus on what should be the asymptotic model in the case of two linearly elastic adherents not perfectly stuck to a thin soft linearly isotropic joint by avoiding lengthy (but not difficult) discussions that occur when the magnitudes of the Lamé coefficients of the joint differ from that of the thickness.

Note that a partial analysis using our method and assorted with numerical experiments may be found in [7] and that our analysis is also valid when the stiffness of the adhesive is not necessarily low but not too high (e.g., less than the inverse of the thickness).

\section{Introducing an elementary situation}

As usual, we make no differences between $\mathbb{R}^{3}$ and the physical Euclidean space whose orthonormal basis is denoted $\left\{e_{1}, e_{2}, e_{3}\right\}$ and, for all $\xi=\left(\xi_{1}, \xi_{2}, \xi_{3}\right) \in \mathbb{R}^{3}, \hat{\xi}$ stands for $\left(\xi_{1}, \xi_{2}\right)$.

Let $\Omega$ be a domain of $\mathbb{R}^{3}$ included in $\left\{x_{3}>0\right\}$ with a Lipschitz boundary $\partial \Omega$. Its intersection with $\left\{x_{3}=0\right\}$ is a domain $S$ of $\mathbb{R}^{2}$, with a positive two-dimensional Hausdorff measure $\mathcal{H}_{2}(S)$, let $B_{\varepsilon}:=S \times(-\varepsilon, 0)$ and $\Omega_{\varepsilon}:=\Omega \cup S \cup B_{\varepsilon}$, where $\varepsilon$ is a small positive number and the boundary of $\Omega_{\varepsilon}$ is assumed to be Lipschitz continuous.

Actually, $\Omega$ is the reference configuration of an elastic body (the adherent), whose bulk energy density, denoted by $W$, is a strictly convex function of the linearized strain tensor $e$, which satisfies

$$
\exists \alpha, \beta>0, \text { s. t. } \alpha|\xi|^{2} \leq W(\xi) \leq \beta\left(1+|\xi|^{2}\right), \forall \xi \in \mathbb{S}^{3 \times 3}
$$

with $\mathbb{S}^{3 \times 3}$ being the space of the $3 \times 3$ symmetric matrices. This is not a gratuitous mathematical generalization of linear isotropic elasticity: bulk energy densities satisfying (1) account for materials with different behavior in tension or compression. They can be obtained ([8], [9, p. 109-117]) by homogenization of micro-cracked linearly elastic media and are good representatives of the macroscopic behavior of concrete, extensively used as adherents in Civil Engineering. The body is clamped on $\Gamma_{0} \subset \partial \Omega \backslash \bar{S}$ and subjected to body forces $f$ and surface forces $\varphi$ on $\Gamma_{1}:=\partial \Omega \backslash\left(\bar{S} \cup \Gamma_{0}\right)$, with $\Gamma_{0}$ assumed to be of positive two-dimensional Hausdorff measures. The set $B_{\varepsilon}$ is the reference configuration of a thin adhesive layer made of an elastic material whose bulk energy density reads as

$$
\left.\begin{array}{l}
W_{\mu_{\mathbb{S}}, \mu_{D}}(\xi):=\mu_{\mathbb{S}} W_{1}(\operatorname{tr}(\xi))+\mu_{D} W_{2}(\operatorname{dev}(\xi)) \\
\operatorname{tr}(\xi):=\xi_{11}+\xi_{22}+\xi_{33,} \operatorname{dev}(\xi):=\xi-\frac{1}{3} \operatorname{tr}(\xi) l
\end{array}\right\}
$$

for all $\xi \in \mathbb{S}^{3 \times 3}$, where $I$ is the identity matrix, $\mu_{\mathbb{S}}$ and $\mu_{D}$ are positive numbers, $W_{1}$ and $W_{2}$ are strictly convex and satisfy

$$
\forall i \in\{1,2\}, \exists \alpha_{i}>0, \exists \beta_{i}>0 \text {, s. t. } \alpha_{i}\left|\xi^{i}\right|^{2} \leq W_{i}\left(\xi^{i}\right) \leq \beta_{i}\left(1+\left|\xi^{i}\right|^{2}\right), \forall\left(\xi^{1}, \xi^{2}\right) \in \mathbb{R} \times \mathbb{S}^{3 \times 3} .
$$

Moreover, we assume that there exist two $\mathcal{C}^{1,1}$ functions $W_{i}^{\infty, 2}, i=1,2$, strictly convex positively homogeneous of degree 2 on $\mathbb{R}$ and $\mathbb{S}^{3 \times 3}$, respectively, such that for each $i=1,2$, there exist positive real numbers $c_{i}, \alpha_{i}^{\infty}$ and $r_{i}$ in $[1,2)$ with

$$
\left.\begin{array}{l}
\left|W_{i}\left(\xi_{i}\right)-W_{i}^{\infty, 2}\left(\xi_{i}\right)\right| \leq c_{i}\left(1+\left|\xi_{i}\right|^{r_{i}}\right), \forall\left(\xi_{1}, \xi_{2}\right) \in \mathbb{R} \times \mathbb{S}^{3 \times 3}, \\
\left(D W_{i}^{\infty, 2}\left(\xi_{i}^{1}\right)-D W_{i}^{\infty, 2}\left(\xi_{i}^{2}\right)\right) \cdot\left(\xi_{i}^{1}-\xi_{i}^{2}\right) \geq \alpha_{i}^{\infty}\left|\xi_{i}^{1}-\xi_{i}^{2}\right|^{2}, \forall\left(\xi_{1}^{\prime}, \xi_{2}^{l}\right) \in \mathbb{R} \times \mathbb{S}^{3 \times 3}, I=1,2,
\end{array}\right\}
$$

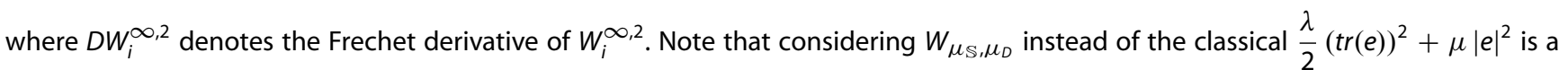
slight generalization of the isotropic linearly elastic case. As the mathematical treatment is similar, this generalization seems to us useful because it may concern materials with different behaviors both in tension and compression and also for hydrostatic and deviatoric straining. This is precisely the case of adhesive bonded joints where the adhesive is filled with fibers or nanoparticles [10] and of the third body in tribology [11]. Actually, there should be minor changes by replacing the growth condition of order 2 by a growth condition of order $p$ in $(1, \infty)$.

The adhesive is clamped along $S_{-\varepsilon}:=-\varepsilon e_{3}+S$ and not subjected to forces. Here, we will consider the case when the mechanical constraint between the adhesive and adherents is not necessarily pure adhesion but is described by a surface energy density $h$ satisfying

$\left(H_{1}\right)$ h is a nonnegative convex lower semi-continuous function in $\mathbb{R}^{3}$ such that $h(0)=0$.

Note that this assumption is satisfied by either a continuous function like $\frac{1}{p}|\cdot|^{p}, 1 \leq p<\infty$ or a nonsmooth function like the indicator function $I_{C}$ of a closed convex subset $C$ of $\mathbb{R}^{3}$ containing 0 . An important practical example of such constraints is when $h(v)=\gamma \Phi_{p}(\hat{v})+l_{E}\left(v_{3}\right)$, where $\gamma$ is a nonnegative number, $E$ is a closed interval of $\mathbb{R}$ containing 0 , and $\Phi_{p}=a_{p}|\cdot|^{p}, a_{p}>0$ if $p \in[1, \infty)$, $\Phi_{\infty}=I_{\mathcal{C}}, \mathcal{C}$ a closed convex subset of $\mathbb{R}^{2}$ containing 0 . The constraint implies bilateral contact when $E=\{0\}$, unilateral contact without penetration and with limited play when $E=[0, \delta], \delta>0$, and unilateral contact without penetration when $E=[0, \infty)$. Moreover, the case $\gamma=0$ indicates that the tangential component of the stress vector always vanishes. When $\gamma \neq 0$, the sole realistic situation is $E=\{0\}$, then the constraint corresponds to bilateral contact with a confined and resistance-free sliding when $p=\infty$ or sliding with Tresca-type resistance when $p=1$ or Norton-Hoff type resistance when $1<p<\infty$. The particular case $p=1$ and $E=\{0\}$ were treated in [6].

In the following, for all domain $G, H_{\gamma}^{1}\left(G ; \mathbb{R}^{3}\right)$ will denote the subspace of the Sobolev space $H^{1}\left(G ; \mathbb{R}^{3}\right)$ whose elements vanish on a smooth enough part $\gamma$ of the boundary $\partial G$ of $G$. 
Hence, if we assume that $(f, \varphi) \in L^{2}\left(\Omega ; \mathbb{R}^{3}\right) \times L^{2}\left(\Gamma_{1} ; \mathbb{R}^{3}\right)$, it is well known that the equilibrium configuration is given by the unique solution $\bar{u}_{s}$ of the following problem involving the triplet of data $s:=\left(\varepsilon, \mu_{S}, \mu_{D}\right)$ :

$$
\left(\mathcal{P}_{s}\right): \operatorname{Min}\left\{F_{s}(v)-L(v) ; v \in V_{s}\right\}
$$

with

$$
\begin{aligned}
v_{s} & :=\left\{v \in L^{2}\left(\Omega_{\varepsilon} ; \mathbb{R}^{3}\right) ; v^{+}:=v_{L \Omega} \in H_{\Gamma_{0}}^{1}\left(\Omega ; \mathbb{R}^{3}\right), v^{-}:=v_{L B_{\varepsilon}} \in H_{S_{-\varepsilon}}^{1}\left(B_{\varepsilon} ; \mathbb{R}^{3}\right)\right\} ; \\
L(v) & :=\int_{\Omega} f(x) \cdot v(x) d x+\int_{\Gamma_{1}} \varphi(x) \cdot v(x) d s, \text { the work of the exterior loading; } \\
{[v] } & \left.:=\gamma_{0}\left(v^{+}\right)-\gamma_{0}\left(v^{-}\right) \text {the jump of displacement across } S \text { (or the relative displacement along } S\right) ; \\
F_{S}(v) & :=\left\{\begin{array}{l}
\int_{\Omega} W\left(e\left(v^{+}\right)\right) d x+\int_{B_{\varepsilon}} W_{\mu_{S}, \mu_{D}}\left(e\left(v^{-}\right)\right) d x+\int_{S} h([v](\hat{x})) d \hat{x}, \text { when } h([v]) \in L^{1}(S), \\
+\infty \text { otherwise. }
\end{array}\right.
\end{aligned}
$$

We use the same symbol $\gamma_{0}(w)$ to denote the trace on $S$ of any element $w$ of both $H^{1}\left(B_{\varepsilon} ; \mathbb{R}^{3}\right)$ and $H^{1}\left(\Omega ; \mathbb{R}^{3}\right)$. To shorten the notations, in the sequel, we will drop the superscript + and - for any element $v$ of $V_{s}$ when no confusion is possible.

Actually, determining numerical approximations of $\bar{u}_{s}$ may be tricky because of the high number of degrees of freedom implied by meshing of the very thin adhesive layer and the ill-conditioned system because of the low stiffness of the glue. Thus, it is of interest to propose a simpler but accurate enough model for the structure. For that purpose, we will consider $s$ as a triplet of small parameters taking values in a countable set of $(0,+\infty)^{3}$ and derive our modeling through a rigorous mathematical study of the asymptotic behavior of $\bar{u}_{s}$ when $s$ goes to its natural limit by taking due account of the low adhesive thickness and stiffness values. Actually, the following analysis works also when $\varepsilon \mu_{\mathbb{S}}$ and $\varepsilon \mu_{D}$ go to zero, that is, also when the stiffness is not too high. Henceforth, we will make the following assumption:

$$
\left\{\begin{array}{l}
\exists \bar{s} \in\{0\} \times[0, \infty]^{2}, \exists\left(\bar{\mu}_{\mathbb{S}}, \bar{\mu}_{D}\right) \in[0, \infty]^{2}, \exists \varepsilon_{0} \in(0, \infty) \text { such that } \\
\bar{s}=\lim s,\left(\bar{\mu}_{\mathbb{S}}, \bar{\mu}_{D}\right)=\left(\lim \mu_{\mathbb{S}} / \varepsilon, \mu_{D} / \varepsilon\right), 0=\lim \left(\varepsilon \mu_{\mathbb{S}}, \varepsilon \mu_{D}\right), 0<\varepsilon<\varepsilon_{0} .
\end{array}\right.
$$

As usual, $c$ or $C$ will denote constants independent of $s$, which may vary from line to line.

\section{An asymptotic model}

The theory initiated in [2] and completed in [1] states that the thin layer is, when $s$ tends to $\bar{s}$, asymptotically equivalent to a mechanical constraint along $S$ whose surface energy density reads as

$$
\bar{W}_{\bar{\mu}_{\mathbb{S}}, \bar{\mu}_{D}}(v)=W_{\bar{\mu}_{\mathbb{S}}, \bar{\mu}_{D}}^{\infty, 2}\left(v \otimes_{s} e_{3}\right):=\bar{\mu}_{\mathbb{S}} W_{1}^{\infty, 2}\left(\operatorname{tr}\left(v \otimes_{s} e_{3}\right)\right)+\bar{\mu}_{D} W_{2}^{\infty, 2}\left(\operatorname{dev}\left(v \otimes_{s} e_{3}\right)\right) \text { for all } v \in \mathbb{R}^{3},
$$

where

$$
a \otimes_{s} b=\frac{1}{2}(a \otimes b+b \otimes a) \text { for all } a, b \in \mathbb{R}^{3} .
$$

Of course when $\bar{\mu}_{\mathbb{S}}$ and $\bar{\mu}_{D}$ are not finite, $\bar{\mu}_{\mathbb{S}} W_{1}^{\infty, 2}$ and $\bar{\mu}_{D} W_{2}^{\infty, 2}$ are replaced by $I_{\{0\}}$. The convention $\infty \times 0=0$ then makes the uniform writing (6) possible. Note that

$$
\left\{\begin{array}{l}
\operatorname{tr}\left(v \otimes_{s} e_{3}\right)=v_{3} \\
\operatorname{dev}\left(v \otimes_{s} e_{3}\right)_{11}=\operatorname{dev}\left(v \otimes_{s} e_{3}\right)_{22}=-\frac{1}{2} \operatorname{dev}\left(v \otimes_{s} e_{3}\right)_{33}=-\frac{v_{3}}{3} \\
\operatorname{dev}\left(v \otimes_{s} e_{3}\right)_{12}=0, \operatorname{dev}\left(v \otimes_{s} e_{3}\right)_{\alpha 3}=\frac{v_{\alpha}}{2}, \alpha=1,2
\end{array}\right.
$$

so that

$$
\bar{W}_{\bar{\mu}_{\mathbb{S}}, \bar{\mu}_{D}}(v) \geq \frac{\alpha_{2}}{2} \bar{\mu}_{D}|v|^{2} \text { for all } v \in \mathbb{R}^{3} .
$$

Thus, we expect to be faced with the connection in series of the initial mechanical constraint with surface energy density $h$ and the

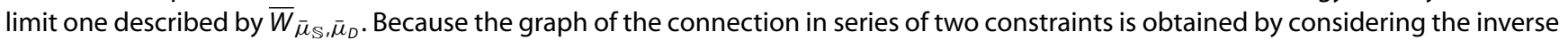
of the addition of the inverse of the graph of each constraint, our asymptotic model should involve the inf-convolution or epigraphical sum $g: h \# \bar{W}_{\bar{\mu}_{\mathbb{S}}, \bar{\mu}_{D}}$ of $h$ and $\bar{W}_{\bar{\mu}_{\mathbb{S}}, \bar{\mu}_{D}}([12])$ defined by

$$
g(t):=h \# \bar{W}_{\bar{\mu}_{\mathbb{S}}, \bar{\mu}_{D}}(t):=\inf \left\{h\left(t^{\prime}\right)+\bar{W}_{\bar{\mu}_{\mathbb{S}}, \bar{\mu}_{D}}\left(t^{\prime \prime}\right) ; t=t^{\prime}+t^{\prime \prime}, t^{\prime}, t^{\prime \prime} \in \mathbb{R}^{3}\right\} .
$$

To deal easily with some singular cases, we introduce the additional assumption on $h$ :

$\left(H_{2}\right)$ When $\left(\bar{\mu}_{\mathbb{S}}, \bar{\mu}_{D}\right) \in\{\infty\} \times(0, \infty)$ or $\left(\bar{\mu}_{\mathbb{S}}, \bar{\mu}_{D}\right) \in(0, \infty] \times\{0\}$, then $h(t)=h_{\wedge}(\hat{t})+h_{3}\left(t_{3}\right)$ for all $t$ in $\mathbb{R}^{3}$ with $h_{\wedge}, h_{3}$ two nonnegative convex lower semicontinuous functions in $\mathbb{R}^{2}$ and $\mathbb{R}$, respectively, and such that $h_{\wedge}(0)=h_{3}(0)=0$, 
which enables us to establish the following properties of $g$ :

Proposition 3.1

The inf-convolution $g=h \# \bar{W}_{\bar{\mu}_{\mathbb{S}}, \bar{\mu}_{D}}$ is a nonnegative convex function defined in $\mathbb{R}^{3}$ such that $g(0)=0$ and

(i) when $\bar{\mu}_{D}=\infty: g=h$,

(ii) when $\bar{\mu}_{D} \in(0, \infty)$ and

(a) $\bar{\mu}_{\mathbb{S}} \in[0, \infty): g$ is continuous on $\mathbb{R}^{3}, g \leq \operatorname{Min}\left(h, C|\cdot|^{2}\right)$; moreover, for all $t$ in $\mathbb{R}^{3}$, there exists a unique $z(t)$ in $\mathbb{R}^{3}$ such that $g(t)=h(t-z(t))+\bar{W}_{\bar{\mu}_{s}, \bar{\mu}_{D}}(z(t))$, with $z$ being a Lipschitz continuous function vanishing at 0

(b) $\bar{\mu}_{\mathbb{S}}=\infty: g(t)$ is finite if and only if $h_{3}\left(t_{3}\right)$ is finite and then $g$ satisfies $g(t) \leq h_{3}\left(t_{3}\right)+C|\hat{t}|^{2}$; moreover, for all $\hat{t}$ in $\mathbb{R}^{2}$, there exists a unique $\hat{z}(\hat{t})$ in $\mathbb{R}^{2}$ such that $g(t)=h_{3}\left(t_{3}\right)+h_{\wedge}(\hat{t}-\hat{z}(\hat{t}))+\bar{\mu}_{D} W_{2}^{\infty, 2}\left(\operatorname{dev}\left((\hat{z}(\hat{t}), 0) \otimes_{s} e_{3}\right)\right)$, with $\hat{z}$ being a Lipschitz continuous function vanishing at 0 , the previous constant $C$ and the Lipschitz continuity constant of $\hat{z}$ being independent of $t_{3}$.

(iii) when $\bar{\mu}_{D}=0$ and

(a) $\bar{\mu}_{\mathbb{S}}=0: g=0$

(b) $\bar{\mu}_{\mathbb{S}} \in(0, \infty): g(t)=\left(h_{3} \# \bar{\mu}_{\mathbb{S}} W_{1}^{\infty, 2}\right)\left(t_{3}\right)$ for all $t \in \mathbb{R}^{3}$, so that $g$ is continuous on $\mathbb{R}^{3}, g(t) \leq \operatorname{Min}\left(h_{3}\left(t_{3}\right), C \bar{\mu}_{\mathbb{S}}\left|t_{3}\right|^{2}\right)$ and there exists a unique $z_{3}\left(t_{3}\right)$ such that $g(t)=h_{3}\left(t_{3}-z_{3}\left(t_{3}\right)\right)+\bar{\mu}_{s} W_{1}^{\infty, 2}\left(z_{3}\left(t_{3}\right)\right)$, with $z_{3}$ being a Lipschitz function vanishing at 0

(c) $\bar{\mu}_{\mathbb{S}}=\infty: g(t)=h_{3}\left(t_{3}\right)$ for all $t \in \mathbb{R}^{3}$.

The proof of this capital proposition (see our proof of Proposition 3.3) is given in Section 4. Hence, we are in a position to define a functional in $H_{\Gamma_{0}}^{1}\left(\Omega ; \mathbb{R}^{3}\right)$ by

$$
F(v):= \begin{cases}\int_{\Omega} W(e(v)) d x+\int_{S} g\left(\gamma_{0}(v)\right) d \hat{x}, & \text { when } g\left(\gamma_{0}(v)\right) \in L^{1}(S), \\ +\infty & \text { otherwise. }\end{cases}
$$

Indeed, to deal with the singular case $\bar{\mu}_{\mathbb{S}}=\bar{\mu}_{D}=0$, hereafter, we make the additional assumption

$$
\left(H_{3}\right) \limsup _{s \rightarrow \bar{s}} \frac{\varepsilon^{3}}{\mu_{\mathbb{S}}}=\limsup _{s \rightarrow \bar{s}} \frac{\varepsilon^{3}}{\mu_{D}}=0 ; \quad \text { if } \bar{\mu}_{\mathbb{S}} \text { or } \bar{\mu}_{D}=0, \exists \alpha_{h}>0, \text { s. t. } h(t) \geq \alpha_{h}(|t|-1), \forall t \in \mathbb{R}^{3},
$$

which is essential, in these singular cases, to establish following Proposition 3.2 that mainly describes the asymptotic behavior of $\left(v_{s}\right)^{-}$ for a sequence such that $F_{s}\left(v_{s}\right) \leq C$. So our asymptotic model will be supplied by the following convergence result:

Theorem 3.1

When $s$ goes to $\bar{s},\left(\bar{u}_{s}\right)+$ strongly converges in $H_{\Gamma_{0}}^{1}\left(\Omega ; \mathbb{R}^{3}\right)$ toward the unique solution $\bar{u}$ of

$(\overline{\mathcal{P}}): \operatorname{Min}\left\{F(v)-L(v) ; v \in H_{\Gamma_{0}}^{1}\left(\Omega ; \mathbb{R}^{3}\right)\right\}$

and $F(\bar{u})=\lim _{s \rightarrow \bar{s}} F_{s}\left(\bar{u}_{s}\right)$.

Actually, this result of variational convergence is a classical consequence of the following three propositions whose proofs can also be found in Section 4:

Proposition 3.2

For all sequences $\left(v_{s}\right)$ in $V_{s}$ such that $F_{s}\left(v_{s}\right) \leq C$, there exists a nonrelabeled subsequence such that

(i) $\left(v_{s}\right)^{+}$weakly converges in $H_{\Gamma_{0}}^{1}\left(\Omega ; \mathbb{R}^{3}\right)$ toward some $v$;

(ii)

(a) $\int_{B_{\varepsilon}}\left|v_{s}\right|^{2} d x \leq C \varepsilon^{2}\left(\frac{1}{\mu_{\mathbb{S}}}+\frac{1}{\mu_{D}}\right), \int_{S}\left|\gamma_{0}\left(\left(v_{s}\right)^{-}\right)\right|^{2} d \hat{x} \leq C \varepsilon\left(\frac{1}{\mu_{\mathbb{S}}}+\frac{1}{\mu_{D}}\right)$;

(b) when $\bar{\mu}_{\mathbb{S}}, \bar{\mu}_{D} \in(0,+\infty], \gamma_{0}\left(\left(v_{s}\right)^{-}\right)$weakly converges in $L^{2}\left(S ; \mathbb{R}^{3}\right)$ toward some $\zeta$, moreover, $\zeta_{3}=0$ if $\bar{\mu}_{S}=\infty, \zeta=0$ if $\bar{\mu}_{D}=\infty$, and when $\bar{\mu}_{\mathbb{S}}=\bar{\mu}_{D}=\infty, \gamma_{0}\left(\left(v_{s}\right)^{-}\right)$converges strongly in $L^{2}\left(S ; \mathbb{R}^{3}\right)$ toward 0 ; when $\bar{\mu}_{\mathbb{S}}=0$ or $\bar{\mu}_{D}=$ $0, \gamma_{0}\left(\left(v_{s}\right)^{-}\right)$weak * converges in the space of bounded measures $M_{b}\left(S ; \mathbb{R}^{3}\right)$ toward some $\zeta ;$

(c) $\int_{-\varepsilon}^{0} \operatorname{tr}\left(e\left(\left(v_{s}\right)\right)\right)\left(., x_{3}\right) d x_{3}$ converges in $L^{2}(S)$ toward $\zeta_{3}=\operatorname{tr}\left(\zeta \otimes_{s} e_{3}\right)$ weakly when $\bar{\mu}_{\mathbb{S}}>0$ and strongly when $\bar{\mu}_{\mathbb{S}}=\infty$, $\int_{-\varepsilon}^{0} \operatorname{dev}\left(e\left(\left(v_{s}\right)\right)\right)\left(., x_{3}\right) d x_{3}$ converges in $L^{2}\left(S ; \mathbb{S}^{3 \times 3}\right)$ toward $\operatorname{dev}\left(\zeta \otimes_{S} e_{3}\right)$ weakly when $\bar{\mu}_{D}>0$ and strongly when $\bar{\mu}_{D}=\infty$, moreover, $\zeta$ belongs to $L^{2}\left(S ; \mathbb{R}^{3}\right)$ when $\left(\bar{\mu}_{\mathbb{S}}, \bar{\mu}_{D}\right) \in\{0\} \times(0, \infty]$ and $\zeta_{3}$ belongs to $L^{2}(S)$ when $\left(\bar{\mu}_{\mathbb{S}}, \bar{\mu}_{D}\right) \in(0, \infty] \times\{0\}$. 
Proposition 3.3

For all $u$ in $H_{\Gamma_{0}}^{1}\left(\Omega ; \mathbb{R}^{3}\right)$, there exists a sequence $\left(u_{s}\right)$ in $V_{s}$ such that $\left(u_{s}\right)^{+}$strongly converges in $H_{\Gamma_{0}}^{1}\left(\Omega ; \mathbb{R}^{3}\right)$ toward $u$ and $F(u) \geq$ $\lim \sup _{s \rightarrow \bar{s}} F_{s}\left(u_{s}\right)$.

$s \rightarrow \bar{s}$

Proposition 3.4

For all $u$ in $H_{\Gamma_{0}}^{1}\left(\Omega ; \mathbb{R}^{3}\right)$ and all sequences $\left(v_{s}\right)$ in $V_{s}$ such that $\left(v_{s}\right)^{+}$weakly converges in $H_{\Gamma_{0}}^{1}\left(\Omega ; \mathbb{R}^{3}\right)$ toward $u$, we have

(i) $J(u):=\int_{\Omega} W(e(u)) d x \leq \liminf _{s \rightarrow \bar{s}} \int_{\Omega} W\left(e\left(v_{s}\right)\right) d x$;

(ii) $\int_{S} g\left(\gamma_{0}(u)\right) d \hat{x} \leq \liminf _{s \rightarrow \bar{s}}\left(\int_{S} h\left(\left[v_{s}\right]\right) d \hat{x}+\int_{B_{\varepsilon}} W_{\mu_{S}, \mu_{D}}\left(e\left(v_{s}\right)\right) d x\right)$;

(iii) $F(u) \leq \liminf _{s \rightarrow \bar{s}} F_{s}\left(v_{s}\right)$.

Thus, Theorem 3.1 describes the asymptotic behavior of the displacement field inside the adherent. Our limit model, problem $(\overline{\mathcal{P}})$, concerns the equilibrium of the elastic adherent subjected to body forces $f$ and surface forces $\varphi$ on $\Gamma_{1}$, clamped along $\Gamma_{0}$ and subjected to a mechanical constraint along $S$ of energy density $g$, which is the inf-convolution of the genuine energy $h$ with the limit surface energy $\bar{W}_{\bar{\mu}_{\mathbb{S}}, \bar{\mu}_{D}}$ stemming from the bulk energy of the thin adhesive layer.

When the adhesive is isotropic and linearly elastic, that is, $W_{1}=\frac{1}{2}|\cdot|^{2}, W_{2}=|\cdot|^{2}, \mu_{\mathbb{S}}=\frac{3 \lambda+2 \mu}{3}, \mu_{D}=\mu$, with $\lambda$ and $\mu$ being the classical Lamé coefficients and $h(t)=h_{\wedge}(\hat{t})+h_{3}\left(t_{3}\right)$ with $h_{3}=I_{\left\{t_{3}=0\right\}}, h_{\wedge}=\frac{a_{p}}{p}|\cdot|^{p}, p \in[1, \infty), h_{\wedge}=I_{\{|\hat{t}| \leq 1\}}$ if $p=\infty$, it is easy to establish that

(i) $\lim \frac{\mu}{\varepsilon}=\infty: g(t)=\frac{a_{p}}{p}|\hat{t}|^{p}+I_{\left\{t_{3}=0\right\}}\left(t_{3}\right)$;

(ii) $\lim \frac{\mu}{\varepsilon}=\bar{\mu} \in(0, \infty)$ :

(a) $\lim \frac{\lambda}{\varepsilon}=\bar{\lambda} \in(0, \infty)$ :

(b) $\lim \frac{\lambda}{\varepsilon}=\infty$ :

$$
\begin{array}{ll}
p=1, & g(t)=\frac{1}{2} \max \left(\bar{\mu}|\hat{t}|^{2}, a_{1}|\hat{t}|\right)+\frac{\bar{\lambda}}{2} t_{3}^{2} ; \\
1<p<\infty, & g=h \# \bar{W}_{\bar{\mu}_{\mathbb{S}}, \bar{\mu}_{D} ;} \\
p=\infty, \quad g(t)=\frac{\bar{\mu}}{2}(\max (|\hat{t}|-1,0))^{2}+\frac{\bar{\lambda}}{2} t_{3}^{2} .
\end{array}
$$

$$
\begin{array}{ll}
p=1, & g(t)=\frac{1}{2} \min \left(\bar{\mu}|\hat{t}|^{2}, a_{1}|\hat{t}|\right)+I_{\left\{t_{3}=0\right\}}\left(t_{3}\right) \\
1<p<\infty, & g=h \# \bar{W}_{\bar{\mu}_{\mathbb{S}}, \bar{\mu}_{D} ;} \\
p=\infty, \quad g(t)=\frac{\bar{\mu}}{2}(\max (|\hat{t}|-1,0))^{2}+I_{\left\{t_{3}=0\right\}}\left(t_{3}\right)
\end{array}
$$

(iii) $\lim \frac{\mu}{\varepsilon}=\bar{\mu}=0$ :
(a) $\lim \frac{\lambda}{\varepsilon}=\bar{\lambda} \in(0, \infty), g(t)=\frac{\bar{\lambda}}{2} t_{3}^{2}$;
(b) $\lim \frac{\lambda}{\varepsilon}=\infty, g(t)=I_{\left\{t_{3}=0\right\}}\left(t_{3}\right)$.

From the mechanical standpoint, it is also interesting to determine the graph of the previous constraints, that is, the relationship between the stress vector $\sigma n$ and the displacement $u$. We denote the stress tensor and the unit normal outward $\Omega$ along $S$ by $\sigma$ and $n$, where of course $n=-e_{3}$, so that the displacement and stress vector have tangential and normal components given by

$$
\sigma_{T}=\sigma n-\left(\sigma_{N}\right) n, \sigma_{N}=\sigma n \cdot n ; u_{T}=u-u_{N} n, u_{N}=u \cdot n .
$$

Hence, the various constraints may also read as

(i) $\lim \frac{\mu}{\varepsilon}=\infty$ :

- $p=1,\left|\sigma_{T}\right| \leq a_{1},\left|\sigma_{T}\right|<a_{1} \Longrightarrow u_{T}=0,\left|\sigma_{T}\right|=a_{1} \Longrightarrow \exists \lambda \geq 0 ; u_{T}=-\lambda \sigma_{T}$;

- $1<p<\infty, \sigma_{T}=-a_{p}\left|u_{T}\right|^{p-2} u_{T}$;

- $p=\infty,\left|u_{T}\right| \leq 1$.

the constraint corresponds to a bilateral contact with sliding through Norton-Hoff-like resistance $(1<p<\infty)$ or Tresca-like $(p=1)$ resistance or a confined sliding $(p=\infty)$; the joint has no effect. 
(ii) $\lim \frac{\mu}{\varepsilon}=\bar{\mu} \in(0, \infty)$ :

- $p=1,\left|\sigma_{T}\right| \leq a_{1},\left|\sigma_{T}\right|<a_{1} \Longrightarrow \sigma_{T}=-2 \bar{\mu} u_{T},\left|\sigma_{T}\right|=a_{1} \Longrightarrow \exists \lambda>0 ; u_{T}=-\lambda \sigma_{T}$;

- $1<p<\infty, u_{T}=\left(\frac{1}{\bar{\mu}}+a_{p}^{1-p^{\prime}}\left|\sigma_{T}\right|^{p^{\prime}-2}\right) \sigma_{T}, \frac{1}{p}+\frac{1}{p^{\prime}}=1$;

- $p=\infty, \sigma_{T}=\bar{\mu} \max \left(\left|u_{T}\right|-1,0\right) \frac{u_{T}}{\left|u_{T}\right|}$;

(a) $\lim \frac{\lambda}{\varepsilon}=\bar{\lambda} \in(0,+\infty)$ :

$\sigma_{N}=-(\bar{\lambda}+2 \bar{\mu}) u_{N}$, the constraint corresponds to an elastic pull-back, nonlinear for the tangential component and linear for the normal one;

(b) $\begin{aligned} \lim \frac{\lambda}{\varepsilon} & =\infty \text { : } \\ u_{N} & =0 \text {, th }\end{aligned}$

$u_{N}^{\varepsilon}=0$, the constraint corresponds to a bilateral contact with sliding through nonlinear resistance.

(iii) $\lim \frac{\mu}{\varepsilon}=0$ :

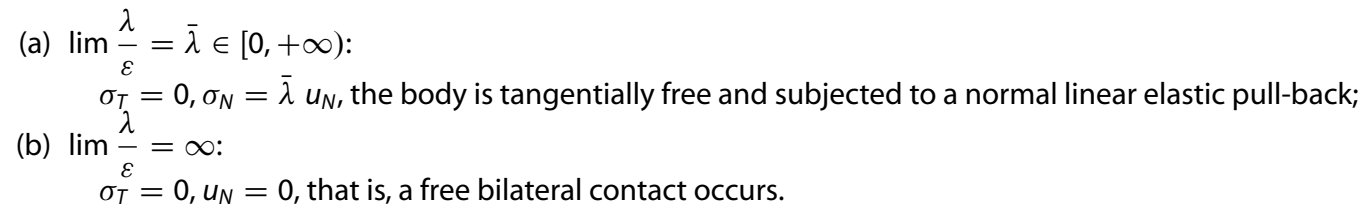

Recall that the laws of case (ii) are used for numerical or theoretical regularization goals [13, 14].

These results were obtained in [6] for $p=1$, but the structure of the density of the limit constraint as an epigraphical sum was not observed. This last point seems important for the mechanical interpretation of the mathematical analysis and its conclusions.

Another realistic example is when $h_{\wedge}=0, h_{3}=I_{[0, T]}, T$ very large which will supply a so-called normal compliance law, which permits a slight penetration in the half-space $\left\{x_{3} \leq 0\right\}$ but with a stiff normal pull-back; it corresponds to the penalization methods in numerical contact mechanics [14].

\section{Remark 3.1}

The coercivity assumption $\left(H_{2}\right)$ on $h$ when $\bar{\mu}_{S}=\bar{\mu}_{D}=0$ was made to obtain boundedness in $L^{2}\left(S, \mathbb{R}^{3}\right)$ of $\gamma_{0}\left(v_{s}^{-}\right)$for any sequence such that $F_{s}\left(v_{s}\right) \leq c$. But this condition prohibits the very frequent condition of unilateral contact where $h_{3}=I_{\left\{t_{3} \geq 0\right\}}$. It has often been observed that the framework of small strains is not suitable to model soft adhesive joints (especially here where $\bar{\mu}_{S}=\bar{\mu}_{D}=0$ suggests very large strains) because it does not take into account interpenetration condition. A first classical remedy is to remain in the framework of small strains, but to include in the formulation of $\left(\mathcal{P}_{s}\right)$ a global interpenetration condition like

$$
\left(\gamma_{0}\left(v^{-}\right)\right)_{3}(\widehat{x}) \geq-\varepsilon \text { for a.e. } \widehat{x} \in S \text {. }
$$

With this additional assumption, the case $h(t)=h_{\wedge}(\hat{t})+I_{\left\{t_{3} \geq 0\right\}}\left(t_{3}\right)$ may be treated because a sequence with bounded $F_{s}\left(v_{s}\right)$ will satisfy

$$
\left(\gamma_{0}\left(\left(v_{s}\right)^{+}\right)\right)_{3} \geq\left(\gamma_{0}\left(\left(v_{s}\right)^{-}\right)\right)_{3} \geq-\varepsilon .
$$

so that the boundedness of $\int_{\Omega}\left|e\left(\left(v_{s}\right)^{+}\right)\right|^{2} d x$ implies the boundedness of $\gamma_{0}\left(\left(v_{s}\right)^{+}\right)_{3}$ in $L^{2}(S)$ and consequently that of $\gamma_{0}\left(\left(v_{s}\right)^{-}\right)_{3}$. Then the limit constraint with energy density $g$ will be the inf-convolution of $h$ and $\bar{W}_{\bar{\mu}_{\mathbb{S}}, \bar{\mu}_{D}}$ augmented by $I_{\left\{t_{3} \geq 0\right\}}$.

It is interesting to improve the modeling by studying the asymptotic behavior of the adhesive layer, which was only suggested in [6]. First, we have

Proposition 3.5

If $\bar{\mu}_{\mathbb{S}}, \bar{\mu}_{D} \in(0, \infty)$, then, when s goes to $\bar{s}, \gamma_{0}\left(\left(\bar{u}_{s}\right)^{-}\right)$weakly converges in $L^{2}\left(S ; \mathbb{R}^{3}\right)$ toward $\bar{\zeta}$ such that



Moreover, if $h$ is strictly convex and satisfies

$$
\exists \alpha_{h}>0, \exists p_{h}>1 \text { s. t. } h(t) \geq \alpha_{h}\left(|t|^{p_{h}}-1\right), \forall t \in \mathbb{R}^{3},
$$

then $\gamma_{0}\left(\left(\bar{u}_{s}\right)^{-}\right)$strongly converges in $L^{q}\left(S ; \mathbb{R}^{3}\right), \forall q \in[1,2)$ if $p_{h}<2, \forall q \in\left[1, p_{h}\right]$ if $2 \leq p_{h}<4, \forall q \in[1,4)$ if $4 \leq p_{h}$.

Furthermore, it is shown in the proof of Proposition 3.3 that there exists $\bar{Z}$ in $H^{1}\left(B_{\varepsilon_{0}} ; \mathbb{R}^{3}\right)$ such that $\bar{\zeta}=\gamma_{0}(\bar{Z})$ and let $R_{\varepsilon} \bar{\zeta} \in$ $H_{S_{-\varepsilon}}^{1}\left(B_{\varepsilon} ; \mathbb{R}^{3}\right)$ be defined by

$$
R_{\varepsilon} \bar{\zeta}(x)=\bar{Z}(x)\left(1+\frac{x_{3}}{\varepsilon}\right) \text { for all } x \in B_{\varepsilon}
$$


Theorem 3.2

If $\bar{\mu}_{\mathbb{S}}, \bar{\mu}_{D} \in(0, \infty)$, then

$$
\lim _{s \rightarrow \bar{s}} \int_{B_{\varepsilon}} W_{\mu_{s}, \mu_{D}}\left(e\left(\left(\bar{u}_{s}\right)^{-}-R_{\varepsilon} \bar{\zeta}\right)\right) d x=\lim _{s \rightarrow \bar{s}} \varepsilon^{-2} \int_{B_{\varepsilon}}\left|\left(\bar{u}_{s}\right)^{-}-R_{\varepsilon} \bar{\zeta}\right|^{2} d x=0
$$

Proposition 3.5 identifies the limit of $\gamma_{0}\left(\left(\bar{u}_{s}\right)^{-}\right)$as the field which achieves the minimum defining the epigraphical sum $g$, while Theorem 3.2 tells us that $\left(\bar{u}_{s}\right)^{-}$is asymptotically equivalent to a field affine in $x_{3}$ with a profile on $S$ precisely given by this limit $\bar{\zeta}$.

Hence, from a practical viewpoint, to easily obtain a good approximation of $\bar{u}_{s}$, we suggest first to solve $(\overline{\mathcal{P}})$ (which is standard from a numerical viewpoint) where $\bar{\mu}_{\mathbb{S}}$ and $\bar{\mu}_{D}$ are replaced by the true real values $\frac{\mu_{\mathbb{S}}}{\varepsilon}$ and $\frac{\mu_{D}}{\varepsilon}$, and to replace $\left(\bar{u}_{s}\right)^{+}$by its solution $\overline{\bar{u}}_{s}$. Next, $\left(\bar{u}_{s}\right)^{-}$may be replaced by $R_{\varepsilon} \bar{\zeta}_{s}$, with $\bar{\zeta}_{s}$ achieving the minimum in the definition of $\left(h \# \bar{W} \frac{\mu_{\mathbb{S}}}{\varepsilon}, \frac{\mu_{D}}{\varepsilon}\right)\left(\gamma_{0}\left(\overline{\bar{u}}_{s}\right)\right)$.

\section{Proofs of the various results}

\subsection{Proof of Proposition 3.1}

The first claim and (i) are obvious [12]. The growth condition in (ii)(a) is obtained by choosing $\left(t^{\prime}, t^{\prime \prime}\right)=(0, t),\left(t^{\prime}, t^{\prime \prime}\right)=(t, 0)$ in definition (8) of $g$ and taking due account of (3). The existence and uniqueness of $z(t)$ for all $t$ in $\mathbb{R}^{3}$ stems from the fact that $h(t-\cdot)+$ $\bar{W}_{\bar{\mu}_{\mathbb{S}}, \bar{\mu}_{D}}$ is a strictly convex coercive function on $\mathbb{R}^{3}$, thus

$$
h\left(t_{j}-z\left(t_{j}\right)\right) \geq h\left(t_{i}-z\left(t_{i}\right)\right)+D \bar{W}_{\bar{\mu}_{\mathbb{S}}, \bar{\mu}_{D}}\left(z\left(t_{i}\right)\right) \cdot\left(t_{j}-z\left(t_{j}\right)-\left(t_{i}-z\left(t_{i}\right)\right)\right), i \neq j \in\{1,2\},
$$

so that (4) yields that there exist positive real numbers $\bar{\alpha}$ and $\bar{\beta}$ such that

$$
\bar{\alpha}\left|z\left(t_{i}\right)-z\left(t_{j}\right)\right|^{2} \leq \bar{\beta}\left|z\left(t_{i}\right)-z\left(t_{j}\right)\right|\left|t_{i}-t_{j}\right| .
$$

The point (ii)(b) is obtained through the same reasoning but with taking due account of $\bar{\mu}_{\mathbb{S}} W_{1}^{\infty, 2}\left(v_{3}\right)=I_{\{0\}}\left(v_{3}\right)$ and of $\left(H_{2}\right)$. Eventually, (iii)(a) is obvious because $\bar{W}_{0,0}=0$, and the splitting assumption $h(t)=h_{\wedge}(\hat{t})+h_{3}\left(t_{3}\right)$ is just made to easily use the previous reasoning in case (b) and to note that $g(t)=\inf \left\{h_{\wedge}(\hat{t}-\hat{v})+h_{3}\left(t_{3}\right) ; \hat{v} \in \mathbb{R}^{2}\right\}$ in case (c).

\subsection{Proof of Proposition 3.2}

Point (i) is an obvious consequence of the coercivity of $W$ and of the Korn inequality. Moreover, if $M_{3}$ denotes the set of all $3 \times 3$ matrices, a Korn inequality like

$$
|\nabla v|_{L^{2}\left(B_{\varepsilon} ; M_{3}\right)} \leq C_{K}|e(v)|_{L^{2}\left(B_{\varepsilon} ; S^{3 \times 3}\right)} \text { for all } v \in H_{S_{-\varepsilon}}^{1}\left(B_{\varepsilon} ; \mathbb{R}^{3}\right)
$$

with $C_{K}$ independent of $\varepsilon$ holds for all $\varepsilon<\varepsilon_{0}$, because it suffices to use the Korn inequality in $H_{S_{-\varepsilon_{0}}}^{1}\left(B_{\varepsilon_{0}}\right)$ for the extension of $v$ by 0 into $B_{\varepsilon_{0}} \backslash B_{\varepsilon}$. Hence, the standard inequality

$$
\int_{B_{\varepsilon}}\left|v_{s}\right|^{2} d x \leq \varepsilon^{2} \int_{B_{\varepsilon}}\left|\partial_{3}\left(v_{s}\right)\right|^{2} d x
$$

implies

$$
\int_{B_{\varepsilon}}\left|v_{s}\right|^{2} d x \leq C_{K}^{2} \varepsilon^{2}\left(\frac{1}{3 \alpha_{1} \mu_{\mathbb{S}}} \int_{B_{\varepsilon}} \mu_{\mathbb{S}} W_{1}\left(\operatorname{tr}\left(e\left(\left(v_{s}\right)\right)\right)\right) d x+\frac{1}{\alpha_{2} \mu_{D}} \operatorname{int}_{B_{\varepsilon}} \mu_{D} W_{2}\left(\operatorname{dev}\left(e\left(\left(v_{s}\right)\right)\right)\right) d x\right)
$$

while the last part of (ii)(a) stems from the other standard one

$$
\int_{S}\left|\gamma_{0}\left(\left(v_{s}\right)^{-}\right)\right|^{2} d \hat{x} \leq \varepsilon \int_{B_{\varepsilon}}\left|\partial_{3}\left(v_{s}\right)\right|^{2} d x
$$

which gives

$$
\int_{S}\left|\gamma_{0}\left(\left(v_{s}\right)^{-}\right)\right|^{2} d \hat{x} \leq C_{K}\left(\frac{\varepsilon}{3 \mu_{\mathbb{S}}} \frac{1}{\alpha_{1}} \int_{B_{\varepsilon}} \mu_{\mathbb{S}} W_{1}\left(\operatorname{tr}\left(e\left(\left(v_{s}\right)\right)\right)\right) d x+\frac{\varepsilon}{\mu_{D}} \frac{1}{\alpha_{2}} \int_{B_{\varepsilon}} \mu_{D} W_{2}\left(\operatorname{dev}\left(e\left(\left(v_{s}\right)\right)\right)\right) d x\right) .
$$

Hence, there exists a nonrelabeled subsequence which converges in $L^{2}\left(S ; \mathbb{R}^{3}\right)$ weakly when $\bar{\mu}_{\mathbb{S}}, \bar{\mu}_{D} \in(0, \infty)$ and strongly to 0 when $\bar{\mu}_{\mathbb{S}}=\bar{\mu}_{D}=\infty$. When $\bar{\mu}_{\mathbb{S}}=\bar{\mu}_{D}=0$, the stated convergence result is true by taking due account of the additional coercivity property $\left(H_{3}\right)$ of $h$ and the previously established point (i).

To complete the proof, it suffices to use the Cauchy-Schwarz inequality and to go to the limit in the identities

$$
\int_{S} p(\hat{x}) \Im^{i} e_{3} \cdot \gamma_{0}\left(\left(v_{s}\right)^{-}\right)(\hat{x}) d \hat{x}=\int_{B_{\varepsilon}} p(\hat{x}) \Im^{i} \cdot e\left(v_{s}\right)(x) d x+\int_{B_{\varepsilon}} \operatorname{div}\left(p \Im^{i}\right) \cdot\left(v_{s}\right)(x) d \hat{x}, i=1-4,
$$

with

$$
\mathfrak{I}^{1}=1, \mathfrak{\Im}^{2}=e_{1} \otimes_{s} e_{3}, \mathfrak{I}^{3}=e_{2} \otimes_{s} e_{3}, \mathfrak{\Im}^{4}=e_{1} \otimes e_{1}+e_{2} \otimes e_{2}-2 e_{3} \otimes e_{3},
$$


$p$ infinitely differentiable with compact support in $S$

and the inequalities

$$
\begin{aligned}
&\left(\int_{B_{\varepsilon}} p \Im^{1} \cdot e\left(v_{s}\right) d x\right)^{2} \leq C(p) \frac{\varepsilon}{\mu_{S}} \frac{1}{\alpha_{1}} \int_{B_{\varepsilon}} \mu_{\mathbb{S}} W_{1}\left(\operatorname{tr}\left(e\left(v_{s}\right)\right)\right) d x \\
&\left(\int_{B_{\varepsilon}} p \Im^{j} \cdot e\left(v_{s}\right) d x\right)^{2} \leq C(p) \frac{\varepsilon}{\mu_{D}} \frac{1}{\alpha_{2}} \int_{B_{\varepsilon}} \mu_{D} W_{2}\left(\operatorname{dev}\left(e\left(v_{s}\right)\right)\right) d x, j=2,4 \\
&\left(\int_{B_{\varepsilon}} \operatorname{div}\left(p \Im^{j}\right) \cdot v_{s} d x\right)^{2} \leq C(p) C_{K}^{2} \varepsilon^{2}\left(\frac{\varepsilon}{3 \mu_{S}} \frac{1}{\alpha_{1}} \int_{B_{\varepsilon}} \mu_{S} W_{1}\left(\operatorname{tr}\left(e\left(v_{s}\right)\right)\right) d x\right. \\
&\left.+\frac{\varepsilon}{\mu_{D}} \frac{1}{\alpha_{2}} \int_{B_{\varepsilon}} \mu_{D} W_{2}\left(\operatorname{dev}\left(e\left(v_{s}\right)\right)\right) d x\right), j=1,4 .
\end{aligned}
$$

\subsection{Proof of Proposition 3.3}

It suffices to consider the case when $F(u)<\infty$. Let us consider the case $\bar{\mu}_{D}=\infty$, we may choose $\left(u_{s}\right)^{+}=u,\left(u_{s}\right)^{-}=0$. Next, when $\left(\bar{\mu}_{D}, \bar{\mu}_{\mathbb{S}}\right) \in(0, \infty) \times[0, \infty)$ we proceed as follows. Any $u$ in $H_{\Gamma_{0}}^{1}\left(\Omega ; \mathbb{R}^{3}\right)$ can be continuously extended in $\mathbb{R}_{+}^{3}:=\left\{x \in \mathbb{R}^{3} ; x_{3}>0\right\}$ by a field $\tilde{u}$ belonging to $H^{1}\left(\mathbb{R}_{+}^{3} ; \mathbb{R}^{3}\right)$. According to point (ii)(a) of Proposition 3.1 and a result of nonlinear interpolation ([15] p. 137), $\zeta:=z(\tilde{u})$ is an element of $H^{1 / 2}\left(S ; \mathbb{R}^{3}\right)$ so that there exists a continuous lifting $Z$ of $\zeta$ into $H_{S_{-\varepsilon_{0}}}^{1}\left(B_{\varepsilon_{0}} ; \mathbb{R}^{3}\right)$. Let $R_{\varepsilon} \zeta$ defined by

$$
R_{\varepsilon} \zeta(x)=Z(x)\left(1+\frac{x_{3}}{\varepsilon}\right), \forall x \in B_{\varepsilon}
$$

Clearly, $R_{\varepsilon} \zeta$ belongs to $H_{S-\varepsilon}^{1}\left(B_{\varepsilon}\right)$ and

$$
\begin{gathered}
e\left(R_{\varepsilon} \zeta\right)(x)=\left(1+\frac{x_{3}}{\varepsilon}\right) e(Z)(x)+Z(x) \otimes_{s} \frac{e_{3}}{\varepsilon}, \forall x \in B_{\varepsilon} \\
\int_{B_{\varepsilon}}\left|R_{\varepsilon} \zeta(x)\right|^{2} d x \leq C\left(\varepsilon \int_{S}|\zeta(\hat{x})|^{2} d \hat{x}+\varepsilon^{2} \int_{B_{\varepsilon}}|\nabla Z(x)|^{2} d x\right)
\end{gathered}
$$

because of

$$
\int_{B_{\varepsilon}}|Z(x)-\zeta(\hat{x})|^{2} d x \leq \varepsilon^{2} \int_{B_{\varepsilon}}|\nabla Z(x)|^{2} d x
$$

Let $u_{s} \in V_{s}$ such that $\left(u_{s}\right)^{+}=u_{1}\left(u_{s}\right)^{-}=R_{\varepsilon} \zeta$. The convexity and the growth conditions (3) satisfied by $W_{1}$ and $W_{2}$ imply [16] that there exist $\gamma_{1}$ and $\gamma_{2}$ such that

$$
\left|W_{i}\left(\xi_{i}^{1}\right)-W_{i}\left(\xi_{i}^{2}\right)\right| \leq \gamma_{i}\left|\xi_{i}^{1}-\xi_{i}^{2}\right|\left(1+\left|\xi_{i}^{1}\right|+\left|\xi_{i}^{2}\right|\right) \forall\left(\xi_{1}^{\prime}, \xi_{2}^{l}\right) \in \mathbb{R} \times \mathbb{S}^{3 \times 3}, I=1,2
$$

and consequently,

$$
\left|W_{i}^{\infty, 2}\left(\xi_{i}^{1}\right)-W_{i}^{\infty, 2}\left(\xi_{i}^{2}\right)\right| \leq \gamma_{i}\left|\xi_{1}^{i}-\xi_{2}^{i}\right|\left(\left|\xi_{1}^{i}\right|+\left|\xi_{2}^{i}\right|\right) \forall\left(\xi_{1}^{l}, \xi_{2}^{l}\right) \in \mathbb{R} \times \mathbb{S}^{3 \times 3}, I=1,2
$$

Hence, the Cauchy-Schwarz inequality gives

$$
\begin{aligned}
& \left|\int_{B_{\varepsilon}}\left(W_{\mu_{S}, \mu_{D}}\left(e\left(u_{s}\right)\right)-W_{\mu_{S}, \mu_{D}}\left(\zeta(\hat{x}) \otimes_{s} \frac{e_{3}}{\varepsilon}\right)\right) d x\right| \\
& \leq C \max \left(\mu_{\mathbb{S}}, \mu_{D}\right)\left\{\int_{B_{\varepsilon}}\left|\left(1+\frac{x_{3}}{\varepsilon}\right) e(Z)+(Z(x)-\zeta(\hat{x})) \otimes s \frac{e_{3}}{\varepsilon}\right|^{2} d x\right\}^{\frac{1}{2}} \\
& \times\left\{\varepsilon+\int_{B_{\varepsilon}}\left(1+\frac{x_{3}}{\varepsilon}\right)^{2}|e(Z)|^{2} d x+\int_{B_{\varepsilon}} \frac{|Z|^{2}(x)}{\varepsilon^{2}} d x+\int_{S} \frac{|\zeta(\hat{x})|^{2}}{\varepsilon} d \hat{x}\right\}^{\frac{1}{2}} \\
& \left.\leq C \max \left(\mu_{S}, \mu_{D}\right)\left(\int_{B_{\varepsilon}}|\nabla Z|^{2} d x\right)^{\frac{1}{2}} \varepsilon+\frac{\int_{S}|\zeta(\hat{x})|^{2} d \hat{x}}{\varepsilon}+\int_{B_{\varepsilon}}|\nabla Z(x)|^{2} d x\right)^{\frac{1}{2}}
\end{aligned}
$$

so that

$$
\begin{aligned}
\lim _{s \rightarrow \bar{s}} \int_{B_{\varepsilon}} W_{\mu_{S}, \mu_{D}}\left(e\left(u_{s}\right)\right) d x & =\lim _{s \rightarrow \bar{s}} \int_{s} W_{\mu_{s}, \mu_{D}}\left(\zeta(\hat{x}) \otimes s \frac{e_{3}}{\varepsilon}\right) d x \\
& =\int_{s} \bar{W}_{\bar{\mu}_{\mathbb{S}}, \bar{\mu}_{D}}(\zeta(\hat{x})) d \hat{x}
\end{aligned}
$$


Thus,

$$
\begin{aligned}
\int_{S} g\left(\gamma_{0}(u)\right) d \hat{x} & =\int_{S}\left(h\left(\gamma_{0}(u)-\zeta\right)+\bar{W}_{\bar{\mu}_{\mathbb{S}}, \bar{\mu}_{D}}(\zeta)\right) d \hat{x} \\
& =\lim _{s \rightarrow \bar{s}}\left(\int_{S} h\left(\left[u_{s}\right]\right) d \hat{x}+\int_{B_{\varepsilon}} W_{\mu_{S}, \mu_{D}}\left(e\left(u_{s}\right)\right) d x\right) \\
\int_{\Omega} W(e(u)) d x & =\lim _{s \rightarrow \bar{s}} \int_{\Omega} W\left(e\left(u_{s}\right)\right) d x .
\end{aligned}
$$

When $\left(\bar{\mu}_{D}, \bar{\mu}_{\mathbb{S}}\right) \in(0, \infty) \times\{\infty\}$, the proof is similar but, now, because of point (ii)(b) of Proposition $3.1,\left(u_{s}\right)^{-}(x)=(\hat{Z}(\hat{x}), 0)\left(1+\frac{x_{3}}{\varepsilon}\right)$ for all $x$ in $B_{\varepsilon}$, where $\hat{Z}$ is a suitable lifting of $\hat{z}\left(\widehat{\gamma_{0}(\widetilde{u})}\right)$.

Eventually, when $\bar{\mu}_{D}=0$, we proceed as previously but, according to point (iii) of Proposition 3.1, with $\hat{\zeta}=\widehat{\gamma_{0}(\tilde{u})}$, and $\zeta_{3}=\gamma_{0}(\tilde{u})_{3}$ when $\bar{\mu}_{\mathbb{S}}=0, \zeta_{3}=z_{3}\left(\gamma_{0}(\tilde{u})_{3}\right)$ when $\bar{\mu}_{\mathbb{S}} \in(0, \infty)$, and $\zeta_{3}=0$ when $\bar{\mu}_{\mathbb{S}}=\infty$.

\subsection{Proof of Proposition 3.4}

The first assertion is a classical consequence of the convexity of $W$. As (iii) is an obvious consequence of (i) and (ii), it remains to prove (ii).

If $\bar{\mu}_{D}=\bar{\mu}_{\mathbb{S}}=0$, each term is simply minorized by 0 because Proposition 3.1 states that $g=0$. In the other cases, we may assume that the infimum limits are finite.

First, if we define $W_{\mu_{\mathbb{S}}, \mu_{D}}^{\infty, 2}$ in the same way as $W_{\bar{\mu}_{\mathbb{S}}, \bar{\mu}_{D}}^{\infty}$ (9) by replacing $\bar{\mu}_{\mathbb{S}}$ and $\bar{\mu}_{D}$ by $\mu_{\mathbb{S}}$ and $\mu_{D}$, then, when $\bar{\mu}_{\mathbb{S},}, \bar{\mu}_{D} \in(0, \infty)$, the Hölder inequality with assumption (4), the Jensen inequality and Proposition 3.2 successively imply

$$
\begin{aligned}
\liminf _{s \rightarrow \bar{s}} \int_{B_{\varepsilon}} W_{\mu_{\mathbb{S}}, \mu_{D}}\left(e\left(v_{s}\right)\right) d x & =\liminf _{s \rightarrow \bar{s}} \int_{B_{\varepsilon}} W_{\mu_{S}, \mu_{D}}^{\infty, 2}\left(e\left(v_{s}\right)\right) d x \\
& \geq \liminf _{s \rightarrow \bar{s}} \int_{S} W_{\bar{\mu}_{\mathbb{S}}, 2, \bar{\mu}_{D}}^{\infty}\left(\int_{-\varepsilon}^{0} e\left(v_{s}\right) d x_{3}\right) d \hat{x} \\
& \geq \int_{S} \bar{W}_{\bar{\mu}_{\mathbb{S}}, \bar{\mu}_{D}}(\zeta) d \hat{x} .
\end{aligned}
$$

To obtain the previous result when one element of $\left\{\bar{\mu}_{S}, \bar{\mu}_{D}\right\}$, say $\bar{\mu}_{\mathbb{S}}$, belongs to $\{0, \infty\}$, we simply have to minorize $W_{\mu_{\mathbb{S}}, \mu_{D}}\left(e\left(v_{S}\right)\right)$ by $\mu_{D} W_{2}\left(\operatorname{dev}\left(e\left(v_{s}\right)\right)\right)$ and use the previous reasoning for $\mu_{D} W_{2}\left(\operatorname{dev}\left(e\left(v_{s}\right)\right)\right)$ instead of $W_{\mu_{S}, \mu_{D}}\left(e\left(v_{s}\right)\right)$, and similarly if $\bar{\mu}_{D}$ belongs to $\{0, \infty\}$. If both $\bar{\mu}_{\mathbb{S}}$ and $\bar{\mu}_{D}$ belong to $\{0, \infty\}$, by Propositions 3.1 and 3.2 , it suffices to minorize by 0 .

Next, when $\bar{\mu}_{D}, \bar{\mu}_{\mathbb{S}} \in(0, \infty]$, Proposition 3.2 and a standard argument of sequential weak $L^{2}\left(S ; \mathbb{R}^{3}\right)$ lower semi-continuity yield

$$
\int_{S} h\left(\gamma_{0}(u)-\zeta\right) d \hat{x} \leq \liminf _{s \rightarrow \bar{s}} \int_{S} h\left(\left[v_{s}\right]\right) d \hat{x} .
$$

If $\bar{\mu}_{\mathbb{S}}=0$, by expressing $h$ in terms of its Legendre-Fenchel transform $h^{*}$, so that

$$
\int_{S} h\left(\left[v_{s}\right]\right) d \hat{x} \geq \int_{S}\left(\gamma_{0}\left(\left(v_{s}\right)^{+}\right)-\gamma_{0}\left(\left(v_{s}\right)^{-}\right) \cdot \varphi(\hat{x}) d \hat{x}-\int_{S} h^{*}(\varphi(\hat{x})) d \hat{x}, \forall \varphi \in C_{0}^{\infty}\left(S ; \mathbb{R}^{3}\right),\right.
$$

then (26) is a mere consequence of Proposition 3.2. If $\bar{\mu}_{D}=0$, by taking into account $\left(H_{1}\right)$ and minorizing $h$ by $h_{3}$, the previous reasoning gives

$$
\int_{S} h_{3}\left(\left(\gamma_{0}(u)-\zeta\right)_{3}\right) d \hat{x} \leq \liminf _{s \rightarrow \bar{s}} \int_{S} h\left(\left[v_{s}\right]\right) d \hat{x}
$$

Thus, (25), (26), and (27) and Proposition 3.1 imply (ii).

\subsection{Proof of Theorem 3.1}

Classically [12], pooling the previous propositions implies all the claims of Theorem 3.1 except the strong convergence in $H_{\Gamma_{0}}^{1}\left(\Omega, \mathbb{R}^{3}\right)$ which (see for instance [17] where such a more or less well-known argument is used) stems from the Korn inequality and the additional fact that $J(\bar{u})=\lim _{s \rightarrow \bar{s}} J\left(\bar{u}_{s}\right)$, the mapping $q \longmapsto \int_{\Omega} W(q) d x$ being strictly convex and coercive on $L^{2}\left(\Omega, \mathbb{S}^{3 \times 3}\right)$. In fact, Proposition 3.4 gives

$$
\begin{aligned}
\limsup _{s \rightarrow \bar{s}} J\left(\bar{u}_{s}\right) & =\limsup _{s \rightarrow \bar{s}}\left(F_{s}\left(\bar{u}_{s}\right)-\left\{\int_{B_{\varepsilon}} W_{\mu_{S}, \mu_{D}}\left(e\left(\bar{u}_{s}\right)\right) d x+\int_{s} h\left(\left[\bar{u}_{s}\right]\right) d \hat{x}\right\}\right) \\
& \leq \lim _{s \rightarrow \bar{s}} F_{s}\left(\bar{u}_{s}\right)-\liminf _{s \rightarrow \bar{s}}\left\{\int_{B_{\varepsilon}} W_{\mu_{S}, \mu_{D}}\left(e\left(\bar{u}_{s}\right)\right) d x+\int_{S} h\left(\left[\bar{u}_{s}\right]\right) d \hat{x}\right\} \\
& \leq F(\bar{u})-\int_{S} g\left(\gamma_{0}(\bar{u})\right) d \hat{x} \\
& =J(\bar{u}) \leq \liminf _{s \rightarrow \bar{s}} J\left(\bar{u}_{s}\right) .
\end{aligned}
$$


4.6. Proof of Proposition 3.5

It has been established in the proof of Proposition 3.4 that

$$
\int_{S}\left(h\left(\gamma_{0}(\bar{u})-\bar{\zeta}\right)+\bar{W}_{\bar{\mu}_{\mathbb{S}}, \bar{\mu}_{D}}(\bar{\zeta})\right) d \hat{x} \leq \liminf _{s \rightarrow \bar{s}}\left(\int_{S} h\left(\left[\bar{u}_{s}\right]\right) d \hat{x}+\int_{B_{\varepsilon}} W_{\mu_{S}, \mu_{D}}\left(e\left(\bar{u}_{s}\right)\right) d x\right),
$$

As

$$
\begin{aligned}
\lim _{s \rightarrow \bar{s}}\left(\int_{S} h\left(\left[\bar{u}_{s}\right]\right) d \hat{x}+\int_{B_{\varepsilon}} W_{\mu_{S}, \mu_{D}}\left(e\left(\bar{u}_{s}\right)\right) d x\right) & =\lim _{s \rightarrow \bar{s}}\left(F_{s}\left(\bar{u}_{s}\right)-J\left(\bar{u}_{s}\right)\right) \\
& =F(\bar{u})-J(\bar{u}) \\
& =\int_{S} g\left(\gamma_{0}(\bar{u})\right) d \hat{x}
\end{aligned}
$$

the first result stems from the very definition of $g$ and Proposition 3.1.

Finally, in the proof of Proposition 3.4, it has also been shown that

$$
\int_{S} \bar{W}_{\bar{\mu}_{s}, \bar{\mu}_{D}}(\bar{\zeta}) d \hat{x} \leq \liminf _{s \rightarrow \bar{s}} \int_{B_{\varepsilon}} W_{\mu_{S}, \mu_{D}}\left(e\left(\bar{u}_{s}\right)\right) d x,
$$

hence,

$$
\begin{aligned}
\limsup _{s \rightarrow \bar{s}} \int_{S} h\left(\left[\bar{u}_{s}\right]\right) d \hat{x} & =\lim _{s \rightarrow \bar{s}}\left(\int_{S} h\left(\left[\bar{u}_{s}\right]\right) d \hat{x}+\int_{B_{\varepsilon}} W_{\mu_{S}, \mu_{D}}\left(e\left(\bar{u}_{s}\right)\right) d x\right)-\liminf _{s \rightarrow \bar{s}} \int_{B_{\varepsilon}} W_{\mu_{\mathbb{S}}, \mu_{D}}\left(e\left(\bar{u}_{s}\right)\right) d x \\
& \leq \int_{S} h\left(\gamma_{0}(\bar{u})-\bar{\zeta}\right) d \hat{x} \\
& \leq \liminf _{s \rightarrow \bar{s}} \int_{S} h\left(\left[\bar{u}_{s}\right]\right) d \hat{x}
\end{aligned}
$$

which establishes the last claim because $\gamma_{0}\left(\left(\bar{u}_{s}\right)^{+}\right)$converges strongly in $L^{q}\left(S, \mathbb{R}^{3}\right), \forall q \in[1,4)$, toward $\gamma_{0}(\bar{u})$ by the Sobolev embeddings.

\subsection{Proof of Theorem 3.2}

Let us consider the scaling $S_{\varepsilon} v$ of any field $v$, defined in $B_{\varepsilon}$, such that

$$
\widehat{S_{\varepsilon} v}\left(\hat{y}, y_{3}\right)=\frac{1}{\varepsilon} \hat{v}\left(\hat{y}, \varepsilon y_{3}\right), \quad\left(S_{\varepsilon} v\right)_{3}\left(\hat{y}, y_{3}\right)=v_{3}\left(\hat{y}, \varepsilon y_{3}\right) \text { for all } y \in B_{1}
$$

and for all $w$ in $H^{1}\left(B_{\varepsilon_{0}} ; \mathbb{R}^{3}\right)$, let $e(\varepsilon, w)$ defined by

$$
e(\varepsilon, w)_{\alpha \beta}=\varepsilon^{2} e_{\alpha \beta}(w), e(\varepsilon, w)_{\alpha 3}=\varepsilon e_{\alpha 3}(w), \alpha, \beta \in\{1,2\} \text { and } e(\varepsilon, w)_{33}=e_{33}(w) .
$$

Arguing as in the second part of the proof of Proposition 3.5 yields

$$
\lim _{s \rightarrow \bar{s}} \int_{B_{\varepsilon}} W_{\mu_{\mathbb{S}}, \mu_{D}}\left(e\left(\bar{u}_{s}\right)\right) d x=\int_{S} \bar{W}_{\bar{\mu}, \mathbb{S}, \bar{\mu}_{D}}(\bar{\zeta}) d \hat{x},
$$

so that (4) implies

$$
\int_{B_{1}} W_{\bar{\mu}_{\mathbb{S}}, \bar{\mu}_{D}}^{\infty, 2}\left(\bar{\zeta} \otimes_{S} e_{3}\right) d y=\lim _{s \rightarrow \bar{s}} \int_{B_{1}} W_{\bar{\mu}_{\mathbb{S}}, \bar{\mu}_{D}}^{\infty}\left(e\left(\varepsilon, S_{\varepsilon} \bar{u}_{s}\right)\right) d y .
$$

Using Proposition 3.2 and 3.4, with $v_{s}:=\theta\left(\bar{u}_{s}\right)^{-}+(1-\theta) R_{\varepsilon} \bar{\zeta}, 0<\theta<1$, and the previous scaling give

$$
\begin{aligned}
\int_{B_{1}} W_{\bar{\mu}_{\mathbb{S}}, \bar{\mu}_{D}}^{\infty, 2}\left(\bar{\zeta} \otimes_{s} e_{3}\right) d y & \leq \liminf _{s \rightarrow \bar{s}} \int_{B_{1}} W_{\bar{\mu}_{\mathbb{S}}, \bar{\mu}_{D}}^{\infty}\left(e\left(\varepsilon, S_{\varepsilon}\left(\theta \bar{u}_{s}+(1-\theta) R_{\varepsilon} \bar{\zeta}\right)\right) d y\right. \\
& \left.=\liminf _{s \rightarrow \bar{s}} \int_{B_{1}} W_{\bar{\mu}_{\mathbb{S}}, \bar{\mu}_{D}}^{\infty, 2}\left(\theta e\left(\varepsilon, S_{\varepsilon} \bar{u}_{s}\right)+(1-\theta) \bar{\zeta} \otimes_{s} e_{3}\right)\right) d y
\end{aligned}
$$

because of (24) and (20)-(22). Hence (28), (29), and the strict convexity of $W_{\bar{\mu}_{s,}, \bar{\mu}_{D}}^{\infty}$ yield that $e\left(\varepsilon, S_{\varepsilon} \bar{u}_{s}\right)$ converges almost everywhere in $B_{1}$ toward $\bar{\zeta} \otimes_{S} e_{3}$ (see Lemma 4.7, the proof of Theorem 4.9, and pages 272-273 in [17] for the details). Now, by using the coercivity of $W_{1}^{\infty, 2}$ and $W_{2}^{\infty, 2}$ (deduced from (3) and (4); Lemma 4.8 page 273 and again the proof of Theorem 4.9 of [17]), we have

$$
\lim _{s \rightarrow \bar{s}} \int_{B_{1}}\left|e\left(\varepsilon, S_{\varepsilon}\left(\bar{u}_{s}\right)\right)-\bar{\zeta} \otimes_{s} e_{3}\right|^{2} d x=0
$$

A change of scale and (20)-(22) give 


$$
\lim _{s \rightarrow \bar{s}} \int_{B_{\varepsilon}} W_{\mu_{\mathbb{S}}, \mu_{D}}\left(e\left(\bar{u}_{s}\right)-R_{\varepsilon} \bar{\zeta}\right) d x=0
$$

while the last assertion stems from (16).

\section{A variant}

A trend in Tribology is to consider that a very thin "third body" is involved during the contact of two deformable bodies [11]. This third body is made from very small parts of matter pulled out from the bodies in the course of the first contacts. Of course, such a third body has a far lower mechanical strength than those of the genuine ones, so the modeling of [1] involving a very soft layer may also be suitable to describe this situation, and complements have been given in [18-20] where dissipative behaviors were treated.

Actually, this trend is improved by considering that in the third body, there are small layers in the vicinity of the bodies in contact with even more downgraded properties. To account for this, we consider the following simplified situation where we assume that a third body lays between an elastic body and a rigid support.

We shall use the notations and assumptions introduced in the previous sections except if explicitly noted. Now, $B_{\varepsilon}$ is the reference configuration of the third body perfectly bonded along $S_{-\varepsilon}$ on a rigid support and perfectly stuck to the elastic body occupying $\Omega$ as reference configuration. We assume that the third body is made of two perfectly bonded elastic parts. The reference configuration of the previously mentioned very small layer inside the third body is $B_{\delta}, 0<\delta<<\varepsilon$, while its bulk energy density is $(2 \delta)^{p-1} k$, where $k$ is a strictly convex function satisfying

$$
\left.\begin{array}{l}
\exists p>1, \exists \alpha_{3}, \beta_{3}>0 ; \alpha_{3}|\xi|^{p} \leq k(\xi) \leq \beta_{3}\left(1+|\xi|^{p}\right) \text { for all } \xi \in \mathbb{S}^{3 \times 3} \\
\exists k^{\infty, p} \text { strictly convex and } p \text {-positively homogeneous such that } \\
\exists r \in[1, p) ;\left|k(\xi)-k^{\infty, p}(\xi)\right| \leq c\left(1+|\xi|^{r}\right) \text { for all } \xi \in \mathbb{S}^{3 \times 3}
\end{array}\right\}
$$

according to [1]; the factor $(2 \delta)^{p-1}$ is chosen so that a limit surface energy $k^{\infty, p}\left(\cdot \otimes_{s} e_{3}\right)$ is supplied.

The reference configuration of the remaining part of the third body is $B_{\varepsilon} \backslash \bar{B}_{\delta}$, and its bulk energy is the density $W_{\mu_{\mathbb{S}}, \mu_{D}}$ introduced in Section 2. Hence, finding the equilibrium position of the structure made of the elastic adherent and the third body, still submitted to the forces $(f, \varphi)$ and clamped along $\Gamma \cup S_{-\varepsilon}$, involves a new parameter $\delta$. Let $\sigma=(s, \delta)=\left(\varepsilon, \mu_{\mathbb{S}}, \mu_{D}, \delta\right)$. We now assume

$$
\left.\begin{array}{l}
\exists \bar{\sigma} \in\{0\} \times[0, \infty]^{2} \times\{0\},\left(\bar{\mu}_{\mathbb{S}}, \bar{\mu}_{D}\right) \in[0, \infty]^{2}, \exists \varepsilon_{0}>0 \text { such that } \\
\bar{\sigma}=\lim \sigma,\left(\bar{\mu}_{\mathbb{S}}, \bar{\mu}_{D}\right)=\lim \left(\frac{\mu_{\mathbb{S}}}{\varepsilon}, \frac{\mu_{D}}{\varepsilon}\right), 0=\lim \left(\varepsilon \mu_{\mathbb{S}}, \varepsilon \mu_{D}\right), 0=\lim \frac{\delta}{\varepsilon}, 0<\varepsilon<\varepsilon_{0} .
\end{array}\right\}
$$

Given the reflexive Banach space $W_{\sigma, p}$ defined by

$$
\left.\begin{array}{c}
p \geq 2, W_{\sigma, p}=\left\{u \in H_{\Gamma_{0} \cup S_{-\epsilon}}^{1}\left(\Omega_{\varepsilon} ; \mathbb{R}^{3}\right) ; e(u) \in L^{p}\left(B_{\delta} ; \mathbb{S}^{3 \times 3}\right)\right\} \\
p<2, W_{\sigma, p}=\left\{u \in W_{\Gamma_{0} \cup S_{-\epsilon}}^{1, p}\left(\Omega_{\varepsilon} ; \mathbb{R}^{3}\right) ; e(u) \in L^{2}\left(\Omega_{\varepsilon} \backslash B_{\delta} ; \mathbb{S}^{3 \times 3}\right)\right\} ; \\
|u|_{\sigma, p}=|e(u)|_{L^{2}\left(\Omega_{\varepsilon} \backslash B_{\delta} ; \mathbb{S}^{3 \times 3}\right)}+|e(u)|_{L^{p}\left(B_{\delta} ; \mathbb{S}^{3 \times 3}\right)}
\end{array}\right\}
$$

and the strictly convex, continuous and coercive function $F_{\sigma}^{\prime}$ on $W_{\sigma, p}$ such that

$$
F_{\sigma}^{\prime}(v)=\int_{\Omega} W(e(v)) d x+(2 \delta)^{p-1} \int_{B_{\delta}} k(e(v)) d x+\int_{B_{\varepsilon} \backslash B_{\delta}} W_{\mu_{\mathbb{S}}, \mu_{D}}(e(v)) d x \text { for all } v \in W_{\sigma, p}
$$

then the problem of finding an equilibrium configuration

$$
\left(\mathcal{P}_{\sigma}^{\prime}\right): \operatorname{Min}\left\{F_{\sigma}^{\prime}(v)-L(v) ; v \in W_{\sigma, p}\right\}
$$

has a unique solution $\bar{u}_{\sigma}^{\prime}$. We aim to study the asymptotic behavior of $\bar{u}_{\sigma}^{\prime}$ when $\sigma$ tends to $\bar{\sigma}$ in order to provide an asymptotic model simpler than $\left(\mathcal{P}_{\sigma}^{\prime}\right)$, where numerical difficulties clearly may occur due to a kind of two-scale meshing.

Because, asymptotically, the energy inside the layer $B_{\delta}$ is equivalent to a surface energy $\int_{s} k^{\infty, p}\left([v] \otimes_{s} e_{3}\right) d \hat{x}$ when $\delta$ goes to zero and $\delta$ is far lower than $\varepsilon$, we guess that we are in a situation close to that studied in Sections 3 and 4, where $h$ will be replaced by $h^{\prime}$

$$
h^{\prime}(t):=k^{\infty, p}\left(t \otimes_{s} e_{3}\right) \text { for all } t \in \mathbb{R}^{3}
$$

That is why, in order to simplify the mathematical analysis, we have considered here that there is only one very thin layer inside the third body. A more realistic situation should involve a second layer occupying $B_{\varepsilon} \backslash B_{\varepsilon}-\delta^{\prime}, 0<\delta^{\prime}<<\varepsilon$, and an analysis similar to the following, but somewhat more technical, may be performed in the spirit of the next section. Hence, we

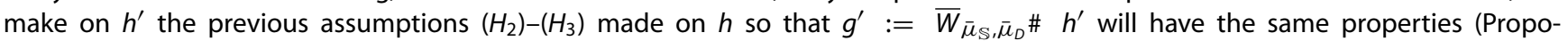
sition 3.1) as $g$; moreover, to simplify the presentation, we also assume $\left(\overline{\mu_{\mathbb{S}}}, \overline{\mu_{D}}\right) \in(0,+\infty)^{2}$, and the asymptotic model is provided by 
Theorem 5.1

When $\sigma$ goes to $\bar{\sigma}, \bar{u}_{\sigma}^{\prime}$ strongly converges in $H_{\Gamma_{0}}^{1}\left(\Omega ; \mathbb{R}^{3}\right)$ toward the unique solution $\bar{u}^{\prime}$ of

where

$$
\left(\mathcal{P}^{\prime}\right): \operatorname{Min}\left\{F^{\prime}(v)-L(v) ; v \in H_{\Gamma_{0}}^{1}\left(\Omega, \mathbb{R}^{3}\right)\right\}
$$

$$
F^{\prime}(v)= \begin{cases}\int_{\Omega} W(e(v)) d x+\int_{S} g^{\prime}\left(\gamma_{0}(v)\right) d \hat{x} & \text { when } g^{\prime}\left(\gamma_{0}(v)\right) \in L^{1}(S) \\ +\infty & \text { otherwise }\end{cases}
$$

As in Section 2, this result of variational convergence is a consequence of the following three propositions:

Proposition 5.1

For all sequences $\left(v_{\sigma}\right)^{+}$in $W_{\sigma, p}$ such that $F_{\sigma}^{\prime}\left(v_{\sigma}\right) \leq C$, there exists a subsequence not relabelled such that

(i) $\left(v_{\sigma}\right)^{+}$weakly converges in $H_{\Gamma_{0}}^{1}\left(\Omega ; \mathbb{R}^{3}\right)$ toward some $v$,

(ii) (a) $\int_{B_{\varepsilon} \backslash B_{\delta}}\left|\left(v_{\sigma}\right)^{-}\right|^{2} d x \leq C \varepsilon^{2}\left(\frac{1}{\mu_{\mathbb{S}}}+\frac{1}{\mu_{D}}\right)$

(b) $\gamma_{0}^{\delta}\left(\left(v_{\sigma}\right)^{-}\right)$, the trace of $\left(v_{\sigma}\right)^{-}$on $S_{-\delta}$ identified with an element of $L^{2}\left(S ; \mathbb{R}^{3}\right)$ weakly converges in $L^{2}\left(S ; \mathbb{R}^{3}\right)$ toward some $\int_{B_{\delta}}\left|v_{\sigma}\right|^{p_{m}} d x \leq C \delta^{\min \left(1, p_{m}-1\right)}$ where $p_{m}=\min (p, 2)$.

Proposition 5.2

For all $u$ in $H_{\Gamma_{0}}^{1}\left(\Omega ; \mathbb{R}^{3}\right)$, there exists a sequence $\left(u_{\sigma}\right)$ in $W_{\sigma, p}$ such that $\left(u_{\sigma}\right)^{+}$strongly converges in $H_{\Gamma_{0}}^{1}\left(\Omega ; \mathbb{R}^{3}\right)$ toward $u$ and $F^{\prime}(u) \geq$ $\limsup _{\sigma \rightarrow \bar{\sigma}} F_{\sigma}^{\prime}\left(u_{\sigma}\right)$.

Proposition 5.3

For all $u$ in $H_{\Gamma_{0}}^{1}\left(\Omega ; \mathbb{R}^{3}\right)$ and all sequences $\left(v_{\sigma}\right)$ in $W_{\sigma, p}$ such that $\left(v_{\sigma}\right)^{+}$weakly converges in $H_{\Gamma_{0}}^{1}\left(\Omega ; \mathbb{R}^{3}\right)$ toward $u$, we have

(i) $J(u):=\int_{\Omega} W(e(u)) d x \leq \liminf _{\sigma \rightarrow \bar{\sigma}} J\left(\left(v_{\sigma}\right)^{+}\right)$,

(ii) $\int_{S} g^{\prime}\left(\gamma_{0}(u)\right) d \hat{x} \leq \liminf _{\sigma \rightarrow \bar{\sigma}}\left(\int_{B_{\varepsilon}}(2 \delta)^{p-1} k\left(e\left(v_{\sigma}\right)\right) d x+\int_{B_{\varepsilon} \backslash B_{\delta}} W_{\mu_{S}, \mu_{D}}\left(e\left(v_{\sigma}\right)\right) d x\right)$,

(iii) $F^{\prime}(u) \leq \liminf _{\sigma \rightarrow \bar{\sigma}} F_{\sigma}^{\prime}\left(v_{\sigma}\right)$.

First, the proofs of Propositions 5.1 and 5.3 follow the lines of those of Propositions 3.2 and 3.4 with $\left(B_{\varepsilon}, S_{-\varepsilon}, S\right)$ replaced firstly by $\left(B_{\varepsilon} \backslash B_{\delta}, S_{-\varepsilon}, S_{-\delta}\right)$ and secondly by $\left(B_{\delta}, S_{-\delta}, S\right)$ while taking due account of the classical inequality

$$
\int_{B_{\delta}}\left|v_{\sigma}\right|^{p_{m}} d x \leq 2^{p_{m}-1}\left(\delta \int_{S}\left|\gamma_{0}\left(v_{\sigma}\right)\right|^{p_{m}} d \hat{x}+\delta^{p_{m}} \int_{B_{\delta}}\left|\nabla v_{\sigma}\right|^{p_{m}} d x\right)
$$

and of

$$
\int_{B_{\delta}}\left|\nabla v_{\sigma}\right|^{p_{m}} d x \leq C \int_{B_{\delta} \cup \Omega}\left|e\left(v_{\sigma}\right)\right|^{p_{m}} d x
$$

derived from the Korn inequality applied to $\min \left(1,1+\frac{x_{3}}{\ell}\right) v_{\sigma}, \ell$ small enough.

Next, to prove Proposition 5.2, it suffices, with respect to the proof of Proposition 3.3, to introduce liftings $U$ and $Z^{\prime}$ of $\gamma_{0}(u)$ and $\zeta^{\prime}$ into $H^{1}\left(B_{\varepsilon_{0}} ; \mathbb{R}^{3}\right), \zeta^{\prime}$ achieving the minimum in the definition of $g^{\prime}\left(\gamma_{0}(u)\right)$ and to define $R_{\varepsilon, \delta}^{\prime}\left(u, \zeta^{\prime}\right)$ by

$$
R_{\varepsilon, \delta}^{\prime}\left(u, \zeta^{\prime}\right):=\left\{\begin{array}{llr}
\left(1+\frac{x_{3}}{\delta}\right) U(x)-\frac{x_{3}}{\delta} Z^{\prime}(x), & \text { if } & 0>x_{3}>-\delta, \\
\left(1+\frac{x_{3}+\delta}{\varepsilon-\delta}\right) Z^{\prime}(x), \& ; \text { if } & -\delta>x_{3}>-\varepsilon .
\end{array}\right.
$$

Then if $\left(u_{\sigma}\right)^{-}=R_{\varepsilon, \delta}^{\prime}\left(u, \zeta^{\prime}\right)$, the arguments used in the proof of Proposition 3.4 work to obtain

$$
\lim _{\sigma \rightarrow \bar{\sigma}} \int_{B_{\varepsilon} \backslash B_{\delta}} W_{\mu_{\mathbb{S}}, \mu_{D}}\left(e\left(u_{s}\right)^{-}\right) d x=\int_{S} \bar{W}_{\bar{\mu}_{\mathbb{S}}, \bar{\mu}_{D}}\left(\zeta^{\prime}\right) d \hat{x}
$$

Moreover, because $e\left(\left(u_{\sigma}\right)^{-}\right)=\left(U-Z^{\prime}\right) \otimes_{s} \frac{e_{3}}{\delta}+\left(1+\frac{x_{3}}{\delta}\right) e(U)-\frac{x_{3}}{\delta} e\left(Z^{\prime}\right)$, the same arguments, but using the convexity and, now, the growth condition of order $p$ for $k$ (see for instance [1]), give

$$
\lim _{\sigma \rightarrow \bar{\sigma}}\left(\int_{B_{\varepsilon} \backslash B_{\delta}}(2 \delta)^{p-1} k\left(e\left(\left(u_{\sigma}\right)^{-}\right)\right) d x\right)=\int_{S} k^{\infty, p}\left(\left(\gamma_{0}(u)-\zeta^{\prime}\right) \otimes_{S} e_{3}\right) d \hat{x}=\int_{S} h^{\prime}\left(\gamma_{0}(u)-\zeta^{\prime}\right) d \hat{x} .
$$


The proof is completed by taking $\left(u_{\sigma}\right)^{+}=u$.

As in Section 3, it is possible to determine the asymptotic behavior of $\bar{u}_{\sigma}^{\prime}$ in the third body

Theorem 5.2

When $\sigma$ goes to $\bar{\sigma}$

(i) $\quad \gamma_{0}^{\delta}\left(\bar{u}_{\sigma}^{\prime}\right)$ weakly converges in $L^{2}\left(S ; \mathbb{R}^{3}\right)$ toward $\bar{\zeta}^{\prime}$ such that



(ii)

$$
\begin{aligned}
& \lim _{\sigma \rightarrow \bar{\sigma}}\left(\int_{B_{\delta}} \delta^{p-1} k\left(e\left(\bar{u}_{\sigma}^{\prime}-R_{\varepsilon, \delta}^{\prime}\left(\bar{u}^{\prime}, \bar{\zeta}^{\prime}\right)\right)\right) d x+\int_{B_{\varepsilon} \backslash B_{\delta}} W_{\mu_{S}, \mu_{D}}\left(e\left(\bar{u}_{\sigma}^{\prime}-R_{\varepsilon, \delta}^{\prime}\left(\bar{u}^{\prime}, \bar{\zeta}^{\prime}\right)\right)\right) d x\right) \\
& =\lim _{\sigma \rightarrow \bar{\sigma}}\left(\delta^{-p} \int_{B_{\delta}}\left|\bar{u}_{\sigma}^{\prime}-R_{\varepsilon, \delta}^{\prime}\left(\bar{u}^{\prime}, \bar{\zeta}^{\prime}\right)\right|^{p} d x+\varepsilon^{-2} \int_{B_{\varepsilon} \backslash B_{\delta}}\left|\bar{u}_{\sigma}^{\prime}-R_{\varepsilon, \delta}^{\prime}\left(\bar{u}^{\prime}, \bar{\zeta}^{\prime}\right)\right|^{2} d x\right)
\end{aligned}
$$

whose proof is similar to those of Proposition 3.5 and Theorem 3.2. Thus, to easily obtain a good approximation of $\bar{u}_{\sigma}^{\prime}$, we first suggest to solve $\left(\mathcal{P}^{\prime}\right)$ where $\bar{\mu}_{\mathbb{S}}$ and $\bar{\mu}_{D}$ are replaced by the true real values $\frac{\mu_{\mathbb{S}}}{\varepsilon}$ and $\frac{\mu_{D}}{\varepsilon}$ and to replace $\left(\bar{u}_{\sigma}^{\prime}\right)^{+}$by its solution $\overline{\bar{u}}_{\sigma}^{\prime}$. Next, $\left(\bar{u}_{\sigma}^{\prime}\right)^{-}$ may be replaced by $R_{\varepsilon, \delta}^{\prime}\left(\overline{\bar{u}}_{\sigma}^{\prime}, \bar{\zeta}_{\sigma}\right), \bar{\zeta}_{\sigma}$ achieving the minimum in the definition of $\left(h^{\prime} \# \bar{W}_{\frac{\mu_{\mathbb{S}}}{\varepsilon}, \frac{\mu_{D}}{\varepsilon}}\right)\left(\gamma_{0}\left(\overline{\bar{u}}_{\sigma}^{\prime}\right)\right)$.

\section{Two adherents imperfectly bonded to a thin joint}

\subsection{Setting out the problem}

The problem of the imperfect bonding of two linearly elastic adherents to a thin soft linearly elastic isotropic joint can be stated as follows. Let $\Omega$ be a domain of $\mathbb{R}^{3}$ with a Lipschitz boundary, with its intersection with $\left\{x_{3}=0\right\}$ being a domain $S$ of $\mathbb{R}^{2}$ with a positive two-dimensional Hausdorff measure. Let $\varepsilon_{0}$ be a positive number and $\Omega^{ \pm}:=\Omega \cap\left\{ \pm x_{3}>0\right\}$; for all $\varepsilon<\varepsilon_{0}$, let $\Omega_{\varepsilon}^{ \pm}:=\Omega^{ \pm} \pm \varepsilon e_{3}$, $\Omega_{\varepsilon}:=\Omega_{\varepsilon}^{+} \cup \Omega_{\varepsilon}^{-}, B_{\varepsilon}:=S \times(-\varepsilon, \varepsilon), S_{\varepsilon}^{ \pm}:=S \pm \varepsilon e_{3}$, then $\Omega_{\varepsilon}^{ \pm}$is the reference configuration of each linearly elastic adherent, while $B_{\varepsilon}$ is that of the adhesive joint. The bulk energy densities of the adherents and of the adhesive are $W$ and $W_{\mu_{S}, \mu_{D}}$, respectively, as in Section 2, and they satisfy (1)-(4). The mechanical contact constraints along $S_{\varepsilon}^{ \pm}$are assumed to be described by two surface energy densities $h^{ \pm}$satisfying the assumptions of Section 2 . If $\Gamma_{0}^{ \pm}$and $\Gamma_{1}^{ \pm}$are two complementary parts of $\partial \Omega^{ \pm} \backslash S$, with $\mathcal{H}_{2}\left(\Gamma_{0}^{+}\right)>0$, we assume that the structure $\mathscr{S}$ made of the two adherents and of adhesive layer is clamped along $\Gamma_{0 \varepsilon}^{ \pm}:=\Gamma_{0}^{ \pm} \pm \varepsilon e_{3}$, subjected to body forces in $\Omega_{\varepsilon}$ only of density $f_{\varepsilon}$ and to surface forces on $\Gamma_{1 \varepsilon}^{ \pm}:=\Gamma_{1}^{ \pm} \pm \varepsilon e_{3}$ of density $\varphi_{\varepsilon}$ where

$$
\begin{aligned}
& f_{\varepsilon}(x)=f\left(\hat{x}, x_{3}+\varepsilon \operatorname{sgn} x_{3}\right), \quad f \in L^{2}\left(\Omega ; \mathbb{R}^{3}\right) \\
& \varphi_{\varepsilon}=g\left(\hat{x}, x_{3}+\varepsilon \operatorname{sgn} x_{3}\right), \quad \varphi \in L^{2}\left(\Gamma_{1}^{ \pm} ; \mathbb{R}^{3}\right) .
\end{aligned}
$$

Thus, finding equilibrium configurations for $\mathcal{S}$ leads to the problem

$$
\left(\mathcal{P}_{s}\right) \quad \min \left\{F_{s}(v)-L_{s}(v): v \in V_{s}\right\}
$$

with

$$
\left\{\begin{array}{l}
V_{s}:=\left\{v \in L^{2}\left(\Omega_{\varepsilon} \cup B_{\varepsilon} ; \mathbb{R}^{3}\right): v^{ \pm}:=v\left\lfloor\Omega_{\varepsilon}^{ \pm} \in H_{\Gamma_{0 \varepsilon}^{ \pm}}^{ \pm}\left(\Omega_{\varepsilon}^{ \pm} ; \mathbb{R}^{3}\right), v^{\prime}:=v\left\lfloor B_{\varepsilon} \in H^{1}\left(B_{\varepsilon} ; \mathbb{R}^{3}\right)\right\}\right.\right. \\
L_{s}(v)=\int_{\Omega_{\varepsilon}} f_{\varepsilon} . v d x+\int_{\Gamma_{1 \varepsilon}^{+} \cup \Gamma_{1 \varepsilon}^{-}} \varphi_{\varepsilon} . v d \mathcal{H}_{2} \\
F_{s}(v):=\left\{\begin{array}{l}
\sum_{ \pm} \int_{\Omega_{\varepsilon}^{ \pm}} W\left(e\left(v^{ \pm}\right)\right) d x+\int_{B_{\varepsilon}} W_{\mu_{S}, \mu_{D}}\left(e\left(v^{\prime}\right)\right) d x+\sum_{ \pm} \int_{S_{\varepsilon}^{ \pm}} h^{ \pm}\left([v]_{\varepsilon}^{ \pm}\right) d \hat{x} \text { when } h^{ \pm}\left([v]_{\varepsilon}^{ \pm}\right) \text {belongs to } L^{1}\left(S_{\varepsilon}^{ \pm}\right)
\end{array}\right.
\end{array}\right.
$$

where $e$ denotes the symmetrical part of the gradient in the sense of distributions of the open sets $\Omega_{\varepsilon}^{ \pm}$and $B_{\varepsilon}$, respectively, and

$$
[v]_{\varepsilon}^{ \pm}:= \pm\left(\gamma_{\varepsilon}^{ \pm}\left(v^{ \pm}\right)-\gamma_{\varepsilon}^{ \pm}\left(v^{\prime}\right)\right)
$$

is the jump of displacement across $S_{\varepsilon}^{ \pm}$(the relative displacement along $S_{\varepsilon}^{ \pm}$)) where the same symbol $\gamma_{\varepsilon}^{ \pm}$stands for the trace operator on $S_{\varepsilon}^{ \pm}$on both $H^{1}\left(\Omega_{\varepsilon}^{ \pm} ; \mathbb{R}^{3}\right)$ and $H^{1}\left(B_{\varepsilon} ; \mathbb{R}^{3}\right)$.

With the additional assumption

$$
\left(H_{4}\right)\left\{\begin{array}{l}
\text { i) } \exists p^{+} \geq 1, \exists \alpha^{+}>0 \text { s.t. } h^{+}(t) \geq \alpha^{+}|t|^{p^{+}} \forall t \in \mathbb{R}^{3} \\
\text { ii) } \mathcal{H}_{2}\left(\Gamma_{0}^{-}\right)>0 \\
\text { or } \\
\text { ii) } \quad \exists p^{-} \geq 1, \exists \alpha^{-}>0 \text { s.t. } h^{-}(t) \geq \alpha^{-}|t|^{p^{-}} \forall t \in \mathbb{R}^{3} \text { and } \int_{\Omega^{-}} \text {f. } \rho d x+\int_{\Gamma_{1}^{-}} \varphi . \rho d \mathcal{H}_{2}=0 \quad \forall \rho \in \mathcal{R},
\end{array}\right.
$$

with $\mathcal{R}$ denoting the space of rigid displacements, we have 
Proposition 6.1

Problem $\mathcal{P}_{s}$ has a unique solution $\bar{u}_{s}$.

The proof is given in Section 6.3. In the following section, we intend to describe the asymptotic behavior of $\bar{u}_{s}$ when $s$ goes to $\bar{s}$.

\subsection{Asymptotic behavior of $\bar{u}_{s}$}

Here, the density associated with the limit mechanical constraint will be defined by

$$
g(t):=\inf \left\{h^{+}\left(t^{+}\right)+\bar{W}_{\bar{\mu}_{\mathbb{S}}, \bar{\mu}_{D}}\left(t^{\prime}\right)+h^{-}\left(t^{-}\right): t^{+}, t^{\prime}, t^{-} \in \mathbb{R}^{3}, t^{+}+t^{\prime}+t^{-}=t\right\}
$$

Note that in this section, $\bar{\mu}_{s}:=\lim \mu_{\mathbb{S}} / 2 \varepsilon, \bar{\mu}_{D}:=\lim \mu_{D} / 2 \varepsilon$. Actually, we will simply indicate what could be the asymptotic modeling in the case of two linearly elastic adherents not perfectly bonded to a thin soft linearly elastic isotropic joint by avoiding lengthly (but not too difficult) discussions that occur when the magnitude of the Lamé coefficients differs from that of the thickness. Moreover, to simplify the discussion of the properties of $g$ and the proof of Proposition 6.4, we make an additional realistic and convenient assumption on the structure of the densities $h^{ \pm}$, and we strengthen assumption $\left(H_{4}\right)$ so that from now on the following is assumed:

$$
\left(H_{5}\right)\left\{\begin{array}{c}
\text { (i) } \bar{\mu}_{D}:=\lim \frac{\mu_{D}}{2 \varepsilon} \in(0,+\infty), \bar{\mu}_{\mathbb{S}}:=\lim \frac{\mu_{\mathbb{S}}}{2 \varepsilon} \in(0,+\infty), \\
\text { (ii) } h^{ \pm}(t)=h_{\wedge}^{ \pm}(\hat{t})+h_{3}^{ \pm}\left(t_{3}\right) \forall t \in \mathbb{R}^{3}, \text { where } h_{\wedge}^{ \pm} \text {satisfies } \\
\quad \exists \alpha^{ \pm}, \beta^{ \pm}>0, \exists p^{ \pm} \geq 1, \alpha^{ \pm}|\hat{t}|^{p^{ \pm}} \leq h_{\wedge}^{ \pm}(\hat{t}) \leq \beta^{ \pm}|t|^{p^{ \pm}} \forall \hat{t} \in \mathbb{R}^{2} \\
\text { and } h_{3}^{ \pm} \text {is the indicator function of a closed bounded interval } /^{ \pm} \text {of } \mathbb{R} \text { containing } 0, \\
\text { (iii) } p^{+}>1 \text { and } \int_{\Omega^{-}} f . \rho d x+\int_{\gamma_{1}^{-}} \varphi \cdot \rho d \mathcal{H}^{2}=0 \forall \rho \in \mathcal{R} \text { when } \mathcal{H}^{2}\left(\Gamma_{0}^{-}\right)=0 .
\end{array}\right.
$$

Hence, $g$ is a well-defined function which obviously satisfies

Proposition 6.2

Function $g$ is a nonnegative strictly convex function in $\mathbb{R}^{3}$ such that

(i) $g(0)=0, g(t) \leq \min \left(C|t|^{2}, h^{ \pm}(t)\right) \forall t \in \mathbb{R}^{3}$ ，

(ii) $\forall t \in \mathbb{R}^{3} \exists !\left(z^{+}(t), z^{\prime}(t), z^{-}(t)\right) \in\left(\mathbb{R}^{3}\right)^{3}$ s.t. $z^{+}(t)+z^{\prime}(t)+z^{-}(t)=t$ and

$$
g(t)=h^{+}\left(z^{+}(t)\right)+\bar{W}_{\bar{\mu}_{\mathbb{S}}, \bar{\mu}_{D}}\left(z^{\prime}(t)\right)+h^{-}\left(z^{-}(t)\right)
$$

To be in a position to state the convergence result, we introduce a kind of translation operator $T_{\varepsilon}$, linear continuous from $H_{\Gamma_{0 \varepsilon}^{+}}^{1}\left(\Omega_{\varepsilon}^{+} ; \mathbb{R}^{3}\right) \times H_{\Gamma_{0 \varepsilon}^{-}}^{1}\left(\Omega_{\varepsilon}^{-} ; \mathbb{R}^{3}\right)$ into $H_{\Gamma_{0}}^{1}\left(\Omega \backslash S ; \mathbb{R}^{3}\right)$ defined for all $u=\left(u^{+}, u^{-}\right)$by

$$
\left(T_{\varepsilon} u\right)(x)=u^{ \pm}\left(x \pm \varepsilon e_{3}\right) \quad \forall x \in \Omega^{ \pm}
$$

then we have

Theorem 6.1

When $s$ goes to $\bar{s}, T_{\varepsilon} \bar{u}_{s}$ strongly converges in $H_{\Gamma_{0}}^{1}\left(\Omega \backslash S ; \mathbb{R}^{3}\right)$ toward the unique solution $\bar{u}$ of

$$
(\overline{\mathcal{P}}) \quad \min \left\{F(v)-L(v): v \in H_{\Gamma_{0}}^{1}\left(\Omega \backslash S ; \mathbb{R}^{3}\right)\right.
$$

and $F(\bar{u})=\lim F_{s}\left(u_{s}\right)$ with

$$
\begin{aligned}
& F(v)=\int_{\Omega} W(e(v)) d x+\int_{s} g([v]) d \hat{x}, \\
& {[v]=\gamma_{0}\left(v^{+}\right)-\gamma_{0}\left(v^{-}\right),} \\
& L(v)=\int_{\Omega} f . v d x+\int_{\Gamma} \varphi . v d \mathcal{H}_{2},
\end{aligned}
$$

with the same symbol $\gamma_{0}$ standing for the trace operator from $H^{1}\left(\Omega^{ \pm} ; \mathbb{R}^{3}\right)$ into $L^{2}\left(S, \mathbb{R}^{3}\right)$.

As in Section 3, this convergence result stems from the following three propositions:

Proposition 6.3

Let $p_{m}^{ \pm}:=\min \left(p^{ \pm}, 2\right)$, then for all sequence $\left(v_{s}\right)$ in $V_{s}$ such that $F_{s}\left(v_{s}\right)-L_{s}\left(v_{s}\right) \leq C$, there exists $v$ in $H_{\Gamma_{0}}^{1}\left(\Omega \backslash S ; \mathbb{R}^{3}\right), \zeta^{ \pm} \in L^{p_{m}^{ \pm}}\left(S ; \mathbb{R}^{3}\right)$ and a nonrelabeled subsequence such that

(i) $T_{\varepsilon} v_{s}$ weakly converges in $H_{\Gamma_{0}}^{1}\left(\Omega \backslash S ; \mathbb{R}^{3}\right)$ toward $v$,

(ii) $\gamma_{\varepsilon}^{+}\left(v_{s}^{\prime}\right)$, identified with an element of $L^{2}\left(S ; \mathbb{R}^{3}\right)$, converges weakly in $L_{m}^{ \pm}\left(S ; \mathbb{R}^{3}\right)$ toward $\zeta^{+}$, $\gamma_{\varepsilon}^{-}\left(v_{s}^{l}\right)$, identified with an element of $L^{2}\left(S ; \mathbb{R}^{3}\right)$, weak * converges in $M_{b}\left(S ; \mathbb{R}^{3}\right)$ when $p^{-}=1$, weakly converges in $L^{p_{m}^{-}}\left(S, \mathbb{R}^{3}\right)$ when $p_{\varepsilon}^{-}>1$, toward $\zeta^{-}$,

(iii) $\int_{-\varepsilon}^{\varepsilon} e\left(v_{s}^{l}\right)\left(., x_{3}\right) d x_{3}$ converges weakly in $L^{2}\left(S, \mathbb{R}^{3}\right)$ toward $\left(\zeta^{+}-\zeta^{-}\right) \otimes_{s} e_{3}$. 
Proposition 6.4

For all $u \in H_{\Gamma_{0}}^{1}\left(\Omega \backslash S ; \mathbb{R}^{3}\right)$, there exists a sequence $\left(u_{s}\right)$ in $V_{s}$ such that $T_{\varepsilon} u_{s}$ weakly converges in $H_{\Gamma_{0}}^{1}\left(\Omega \backslash S ; \mathbb{R}^{3}\right)$ toward $u$ and $F(u) \geq$ $\lim \sup F_{s}\left(u_{s}\right)$.

Proposition 6.5

For all $u \in H_{\Gamma_{0}}^{1}\left(\Omega \backslash S ; \mathbb{R}^{3}\right)$ and all sequences $\left(v_{s}\right)$ in $V_{s}$ such that $T_{\varepsilon} v_{s}$ weakly converges in $H_{\Gamma_{0}}^{1}\left(\Omega \backslash S ; \mathbb{R}^{3}\right)$ toward $u$, we have

(i) $J(u):=\int_{\Omega} W(e(u)) d x \leq \liminf \int_{\Omega_{\varepsilon}} W\left(e\left(v_{s}\right)\right) d x$;

(ii) $\int_{S} g([u]) d \hat{x} \leq \liminf \left(\int_{S_{\varepsilon}^{ \pm}} h^{ \pm}\left(\left[v_{s}\right]_{\varepsilon}^{ \pm}\right) d \hat{x}+\int_{B_{\varepsilon}} W_{\mu_{S}, \mu_{D}}\left(e\left(v_{s}^{\prime}\right)\right) d x\right)$;

(iii) $F\left(u \leq \liminf F_{s}\left(v_{s}\right)\right.$.

Theorem 6.1 describes the asymptotic behavior of the displacement field inside the adherents. Problem $(\overline{\mathcal{P}})$ concerns the equilibrium of the elastic adherents occupying $\Omega^{ \pm}$subjected to body forces $f$ and surface forces $\varphi$ on $\Gamma_{1}$, clamped along $\Gamma_{0}$ and linked by a mechanical constraint along $S$ of energy density $g$, which corresponds to the connection in series of the constraints of densities $h^{ \pm}$and that stemming from the limit behavior of the adhesive joint.

To be in a position to propose a simplified but accurate enough model for the structure $\mathscr{S}$, we also study the asymptotic behavior of the adhesive. The following result can be established similarly to Proposition 3.5 and Theorem 3.2.

Theorem 6.2

When $s$ goes to $\bar{s}$, then

(i) $\gamma_{\varepsilon}^{ \pm}\left(\bar{u}_{s}^{\prime}\right)$ weakly converges in $L^{2}\left(S ; \mathbb{R}^{3}\right)$ toward $\zeta^{ \pm}$such that $g([\bar{u}])=h^{ \pm}\left( \pm\left(\gamma_{0}\left(\bar{u}^{ \pm}\right)-\zeta^{ \pm}\right)+\bar{W}_{\bar{\mu}_{\mathbb{S}}, \bar{\mu}_{D}}\left(\zeta^{+}-\zeta^{-}\right)\right.$, moreover, if $h^{ \pm}$is strictly convex, $\gamma_{\varepsilon}^{ \pm}\left(\overline{u_{s}^{\prime}}\right)$ converges strongly in $L^{q}\left(S ; \mathbb{R}^{3}\right)$ for all $q \in[1,2)$;

(ii) $\lim \int_{B_{\varepsilon}} W_{\mu_{\mathbb{S},} \mu_{D}}\left(e\left(u_{s}^{\prime}-R_{\varepsilon}\left(\zeta^{+}-\zeta^{-}\right)\right) d x=\lim \varepsilon^{-2} \int_{B_{\varepsilon}} \int_{B_{\varepsilon}}\left|u_{s}^{\prime}-R_{\varepsilon}\left(\zeta^{+}-\zeta^{-}\right)\right|^{2} d x\right.$ where $R_{\varepsilon}\left(\zeta^{+}-\zeta^{-}\right)(x)=\frac{1}{2}\left[z^{+}\left(\zeta^{+}\right)\left(\hat{x}_{1}\left|x_{3}\right|\right)+z^{-}\left(\zeta^{-}\right)\left(\hat{x}_{1}-\left|x_{3}\right|\right)\right]+\frac{x_{3}}{\varepsilon}\left[z^{+}\left(\zeta^{+}\right)\left(\hat{x}_{1}\left|x_{3}\right|\right)-z^{-}\left(\zeta^{-}\right)\left(\hat{x}_{,}-\left|x_{3}\right|\right)\right], \forall x \in B_{\varepsilon}$, with $z^{ \pm}\left(\zeta^{ \pm}\right)$being a continuous lifting of $\zeta^{ \pm}$into $H_{s_{\varepsilon_{0}}}^{1}\left(B \frac{ \pm}{\varepsilon_{0}} ; \mathbb{R}^{3}\right), B_{\varepsilon_{0}}^{ \pm}=B_{\varepsilon_{0}} \cap\left\{ \pm x_{3}>0\right\}$.

Theorem 6.2 identifies the limit of $\gamma_{\varepsilon}^{ \pm}\left(\overline{u_{s}^{\prime}}\right)$ as the fields which achieve the infimum, defining the epigraphical sum $g$ and state that $\overline{u_{s}^{\prime}}$ is asymptotically equivalent to a field affine in $x_{3}$ with a profile on $S_{\varepsilon}^{ \pm}$given by $\zeta^{ \pm}$.

Hence, in practice, to easily obtain a good approximation of the behavior of $\bar{u}$, we first suggest to consider the solution $\overline{\bar{u}}$ of $(\overline{\mathcal{P}})$ where $\bar{\mu}_{\mathbb{S}}$ and $\bar{\mu}_{D}$ are replaced by the true real values $\mu_{\mathbb{S}} / 2 \varepsilon$ and $\mu_{D} / 2 \varepsilon$ and next to replace $\bar{u}_{s}$ by $\overline{\bar{u}}_{s}$ defined by

$$
\begin{aligned}
& \overline{\bar{u}}_{s}^{ \pm}\left(\hat{x}, x_{3}\right)=\overline{\bar{u}}\left(\hat{x}, x_{3} \mp \varepsilon\right) \forall x \in \Omega_{\varepsilon}^{ \pm}, \\
& \overline{\bar{u}}_{s}^{I}=R_{\varepsilon}\left(\zeta_{s}^{+}-\zeta_{s}^{-}\right) \text {in } B_{\varepsilon},
\end{aligned}
$$

$\zeta_{s}$ achieving the infimum in the definition of $g([\overline{\bar{u}}])$ but where $\bar{\mu}_{\mathbb{S}}$ and $\bar{\mu}_{D}$ are replaced by $\mu_{\mathbb{S}} / 2 \varepsilon$ and $\mu_{D} / 2 \varepsilon$.

\subsection{Proof of the previous results}

6.3.1. Proof of Proposition 6.1. Clearly, $F_{s}-L_{s}$ is a strictly convex and lower semi-continuous function on $V_{s}$, where

$$
|v|_{s}^{2}:=\int_{\Omega_{\varepsilon}^{ \pm}}|e(v)|^{2} d x+\int_{B_{\varepsilon}}|e(v)|^{2} d x+\int_{S_{\varepsilon}^{ \pm}}\left|[v]_{\varepsilon}^{ \pm}\right|^{2} d \hat{x}
$$

turns it into a Hilbert space (when $\mathcal{H}_{2}\left(\Gamma_{0}^{-}\right)>0$ the integral on $S_{\bar{\varepsilon}}^{-}$may be omitted). But as a quadratic growth for $h^{ \pm}$is too restrictive from the mechanical standpoint, the result is obtained by directly proving that any sequence $\left(v_{n}\right)$ in $V_{s}$ such that $F_{s}\left(v_{n}\right)-L_{s}\left(v_{n}\right) \leq C$ does satisfy

There exists a nonrelabeled subsequence which converges weakly in $V_{s}$ toward some $v$.

Obviously, $v_{n}^{+}$and $v_{n}^{\prime}$ are bounded in $H^{1}\left(\Omega_{\varepsilon}^{+} ; \mathbb{R}^{3}\right)$ and $H^{1}\left(B_{\varepsilon} ; \mathbb{R}^{3}\right) / \mathcal{R}$, while assumption $\left(H_{4}\right)\left(\right.$ ii) or $\left(H_{4}\right)(\text { ii) })^{\prime}$ implies that $v_{n}^{-}$is bounded in $H^{1}\left(\Omega_{\varepsilon}^{-} ; \mathbb{R}^{3}\right)$ or in $H^{1}\left(\Omega_{\varepsilon}^{-} ; \mathbb{R}^{3}\right) / \mathcal{R}$. Consequently, there exist a nonrelabeled subsequence $\left(v_{n}\right)$ in $V_{s}$ and a sequence $\left(\rho_{n}^{\prime}, \rho_{n}^{-}\right)$in $\mathcal{R}^{2}$ with $\rho_{n}^{-}=0$ in case $\left(H_{4}\right)($ ii), such that

$-v_{n}^{+}$converges weakly in $H^{1}\left(\Omega_{\varepsilon}^{+} ; \mathbb{R}^{3}\right)$ toward $v^{+}, \gamma_{\varepsilon}^{+}\left(v_{n}^{+}\right)$converges strongly in $L^{2}\left(S_{\varepsilon}^{+} ; \mathbb{R}^{3}\right)$ toward $\gamma_{\varepsilon}^{+}\left(v^{+}\right)$,

$-\left(v_{n}^{\prime}, v_{n}^{-}\right)+\left(\rho_{n}^{l}, \rho_{n}^{-}\right)$converges weakly in $H^{1}\left(B_{\varepsilon} ; \mathbb{R}^{3}\right) \times H^{1}\left(\Omega_{\varepsilon}^{-} ; \mathbb{R}^{3}\right)$ toward $\left(w^{l}, w^{-}\right)$, and $\left(\gamma_{\varepsilon}^{ \pm}\left(v_{n}^{\prime}+\rho_{n^{\prime}}^{l}\right), \gamma_{\varepsilon}^{-}\left(v_{n}^{-}+\rho_{n}^{-}\right)\right)$converges strongly in $L^{2}\left(S_{\varepsilon}^{ \pm} ; \mathbb{R}^{3}\right) \times L^{2}\left(S_{\varepsilon}^{-} ; \mathbb{R}^{3}\right)$ toward $\left(\gamma_{\varepsilon}^{ \pm}\left(w^{\prime}\right), \gamma_{\varepsilon}^{-}\left(w^{-}\right)\right)$.

Assumption $\left(H_{4}\right)(\text { ii) })^{\prime}$ yields that for a nonrelabeled subsequence of the previous one, $\gamma_{\varepsilon}^{ \pm}\left(v_{n}^{\prime}\right)$ weak $\star$ converges in $M_{b}\left(S_{\varepsilon}^{+} ; \mathbb{R}^{3}\right)$; hence, $\rho_{n}^{l}=v_{n}^{l}+\rho_{n}^{l}-v_{n}^{l}$ weak $\star$ converges in $M_{b}\left(S_{\varepsilon}^{+}, \mathbb{R}^{3}\right)$ and also strongly in $L^{2}\left(B_{\varepsilon} ; \mathbb{R}^{3}\right)$, because $\mathcal{R}$ is finite dimensional. Therefore, there exists $v^{l}$ in $H^{1}\left(B_{\varepsilon}, \mathbb{R}^{3}\right)$ such that $v_{n}^{l}$ converges weakly in $H^{1}\left(B_{\varepsilon} ; \mathbb{R}^{3}\right)$ toward $v^{\prime}$, and $\gamma_{\varepsilon}^{+}\left(v_{n}^{\prime}\right)$ converges strongly in $L^{2}\left(S_{\varepsilon}^{+} ; \mathbb{R}^{3}\right)$ toward $\gamma_{\varepsilon}^{+}\left(v^{\prime}\right)$. Thus, nothing more has to be performed to establish (39) in the case $\left(H_{4}\right)(\mathrm{ii})^{\prime}$.

Assumption $\left(H_{4}\right)$ (ii)' implies that a nonrelabeled subsequence of the previous ones weak $\star$ converges in $M_{b}\left(S_{\varepsilon}^{-} ; \mathbb{R}^{3}\right)$ at least, and we obtain the expected result by reproducing the same previous reasoning involving rigid displacements and taking due account of the last part of $\left(H_{4}\right)(\mathrm{ii})^{\prime}$. 
6.3.2. Proof of Proposition 6.3. First point (i) is obvious when $\mathcal{H}_{2}\left(\Gamma_{0}^{-}\right)>0$ by using the Korn inequality in $\Omega^{+}$and $\Omega^{-}$. Anyway, because of the second part of $\left(H_{5}\right)$ (iii), there exists a sequence $\rho_{s}^{-}$in $\mathcal{R}\left(\rho^{-}=0\right.$ when $\left.\mathcal{H}_{2}\left(\Gamma_{0}^{-}\right)>0\right)$ such that, up to a subsequence, $T_{\varepsilon}\left(v_{s}^{+}, v_{s}^{-}+\rho_{s}^{-}\right)$weakly converges to some $\bar{v}$ in $H_{\Gamma_{0}}\left(\Omega \backslash S ; \mathbb{R}^{3}\right)$ and consequently such that $\gamma_{0}\left(T_{\varepsilon}\left(v_{s}^{+}, v_{s}^{-}+\rho_{s}^{-}\right)\right)$strongly converges in $L^{2}\left(S ; \mathbb{R}^{3}\right)$ toward $\gamma_{0}\left(\bar{v}^{ \pm}\right)$. The additional assumption $p^{+}>1$ in $\left(H_{5}\right)\left(\right.$ iii) implies that, for a nonrelabeled subsequence, $\gamma_{\varepsilon}^{+}\left(v_{s}^{l}\right)$ weakly converges in $L^{p_{m}^{+}}\left(S ; \mathbb{R}^{3}\right)$ toward some $\zeta^{+}$and consequently (we recall that $\bar{\mu}_{\mathbb{S}}, \bar{\mu}_{D} \in(0,+\infty)$ ):

$$
\begin{gathered}
\int_{B_{\varepsilon}}\left|\left(v_{s}^{l}\right)_{3}\right|^{p_{m}^{+}} d x \leq \varepsilon \int_{S_{\varepsilon}}\left|\left(v_{s}^{\prime}\right)_{3}\right|^{p_{m}^{+}} d \hat{x}+\varepsilon^{p_{m}^{+}} \int_{B_{\varepsilon}}\left|e\left(v_{s}^{l}\right)\right|^{p_{m}^{+}} d x \leq C \varepsilon, \\
\int_{S}\left|\int_{-\varepsilon}^{\varepsilon} e\left(v_{s}^{l}\right) d x_{3}\right|^{2} d \hat{x} \leq 2 \varepsilon \int_{B_{\varepsilon}}\left|e\left(v_{s}^{l}\right)\right|^{2} d x \leq C .
\end{gathered}
$$

Hence, in (41) there exists a nonrelabeled subsequence such that $\int_{-\varepsilon}^{\varepsilon} e\left(v_{s}^{l}\right)\left(., x_{3}\right) d x_{3}$ converges weakly in $L^{2}\left(S ; \mathbb{S}^{3 \times 3}\right)$ toward some $e^{\prime}$, which can be identified as to be of the form $e^{\prime}=\left(\zeta^{+}-\zeta^{-}\right) \otimes_{s} e_{3}$ as follows. First, going to the limit on the identity

$$
\int_{S}\left(\int_{-\varepsilon}^{\varepsilon} e\left(v_{s}\right) d x_{3}\right) \cdot \theta(\hat{x}) \tau_{i} d \hat{x}=-\int_{B_{\varepsilon}} v_{s}^{i} \cdot \operatorname{div}\left(\theta \tau_{i}\right) d x+\int_{S}\left(\gamma_{\varepsilon}^{+}\left(v_{s}^{\prime}\right)-\gamma_{\varepsilon}^{-}\left(v_{s}^{\prime}\right)\right) \cdot \theta \tau^{i} e_{3} d \hat{x}
$$

$\tau^{i}:=e_{i} \otimes_{s} e_{3}, i=1,2,3, \forall \theta \in \mathcal{C}_{c}^{\infty}(S)$, where $\gamma_{\varepsilon}^{ \pm}\left(v_{s}^{\prime}\right)$ are identified with an element of $L^{2}\left(S ; \mathbb{R}^{3}\right)$, and by using (40), one deduces that $\gamma_{\varepsilon}^{+}\left(v_{s}^{l}\right)-\gamma_{\varepsilon}^{-}\left(v_{s}^{l}\right)$ has a limit $\zeta^{+}-\zeta^{-}$in the sense of distributions which belong to $L^{2}\left(S ; \mathbb{R}^{3}\right)$, and such that $e_{i 3}^{l}=\left(\zeta^{+}-\zeta^{-}\right)_{i}, i=1,2,3$. Next, as

$$
\int_{-\varepsilon}^{\varepsilon} e_{\alpha \beta}\left(v_{s}^{\prime}\right) d x_{3}=e_{\alpha \beta}\left(\int_{-\varepsilon}^{\varepsilon} v_{s}^{\prime} d x_{3}\right)
$$

we simply have to establish that $\int_{-\varepsilon}^{\varepsilon} v_{s}^{\prime} d x_{3}$ converges to zero in the sense of distributions. The trick consists of combining the identity

$$
\partial_{3}\left(v_{s}^{\prime}\right)_{\alpha}=2 e_{\alpha \beta}\left(v_{s}^{l}\right)-\partial_{\alpha}\left(v_{s}^{\prime}\right)_{3}
$$

and an integration by part in the identity

$$
\int_{S} \theta(\hat{x})\left(\int_{-\varepsilon}^{\varepsilon}\left(v_{s}^{l}\right)_{\alpha} d x_{3}\right) d \hat{x}=2 \varepsilon \int_{S} \theta \gamma_{\varepsilon}^{+}\left(\left(v_{s}^{l}\right)_{\alpha}\right) d \hat{x}+\int_{S} \theta(\hat{x}) \int_{-\varepsilon}^{\varepsilon}\left(\int_{\varepsilon}^{x_{3}} \partial_{3}\left(v_{s}^{l}\right)_{\alpha} d t\right) d x_{3} d \hat{x} .
$$

which then becomes

$$
\int_{S} \theta(\hat{x})\left(\int_{-\varepsilon}^{\varepsilon}\left(v_{s}^{\prime}\right)_{\alpha} d x_{3}\right) d \hat{x}=2 \varepsilon \int_{S} \theta \gamma_{\varepsilon}^{+}\left(\left(v_{s}^{\prime}\right)_{\alpha}\right) d \hat{x}+\int_{S} \theta(\hat{x}) \int_{-\varepsilon}^{\varepsilon}\left(\int_{\varepsilon}^{x_{3}} 2 e_{\alpha \beta}\left(v_{s}^{\prime}\right) d t\right) d x_{3} d \hat{x}+\int_{S} \partial_{\alpha} \theta(\hat{x}) \int_{-\varepsilon}^{\varepsilon}\left(\int_{\varepsilon}^{x_{3}}\left(v_{s}^{\prime}\right)_{3} d t\right) d x_{3} d \hat{x} .
$$

The first term in the right hand arm clearly tends to zero, and according to the Hölder inequality and (41) and (40), respectively, each of the last two terms tend to be zero.

Finally, when $\mathcal{H}_{2}\left(\Gamma_{0}^{-}\right)=0$, we deduce that $\gamma_{0}\left(\left(T_{\varepsilon}\left(v_{s}^{+}, v_{s}^{-}\right)\right)^{-}\right)$converges in the sense of distributions so that $T_{\varepsilon}\left(0, \rho_{s}^{-}\right)^{-}$converges in the sense of distributions and consequently strongly converges in $L^{2}\left(S, \mathbb{R}^{3}\right)$. Therefore, $\gamma_{0}\left(\left(T_{\varepsilon}\left(v_{s}^{+}, v_{s}^{-}\right)\right)^{-}\right)$converges strongly in $L^{2}\left(S, \mathbb{R}^{3}\right)$, which implies that $T_{\varepsilon}\left(v_{s}^{+}, v_{s}^{-}\right)^{-}$converges weakly in $H^{1}\left(\Omega^{-} ; \mathbb{R}^{3}\right)$ and $\left.\gamma_{\varepsilon}^{-}\left(v_{s}^{\prime}\right)\right)$ weak $\star$ in $M_{b}\left(S ; \mathbb{R}^{3}\right)$ and, of course, possibly weakly in $L_{m}^{+}\left(S, \mathbb{R}^{2}\right)$ if $p^{-}>1$.

6.3.3. Proof of Proposition 6.4. If $F_{s}\left(u_{s}\right)<+\infty$, there exists $\zeta^{ \pm}$in $L^{p_{m}^{ \pm}}\left(S, \mathbb{R}^{3}\right)$ such that

$$
\int_{S} g([u]) d \hat{x}=\int_{S} h^{+}\left(\gamma_{0}\left(u^{+}\right)-\zeta^{+}\right) d \hat{x}+\int_{S} \bar{W}_{\bar{\mu}_{S}, \bar{\mu}_{D}}\left(\zeta^{+}-\zeta^{-}\right) d \hat{x}+\int_{S} h^{-}\left(\zeta^{-}-\gamma_{0}\left(u^{-}\right)\right) d \hat{x} .
$$

For any $\eta \geq 0$ let $\xi_{\eta}^{ \pm}$in $\mathcal{C}_{0}^{\infty}\left(S ; \mathbb{R}^{3}\right)$ be such that $\left|\xi_{\eta}^{ \pm}-\zeta^{ \pm}\right|_{L^{p_{m}^{ \pm}}\left(s, \mathbb{R}^{3}\right)} \leq \eta$ and $\zeta_{\eta}$ defined by $\hat{\zeta}_{\eta}^{ \pm}=\hat{\xi}_{\eta}^{ \pm}$, while $\left(\zeta_{\eta}^{ \pm}\right)_{3}$ is the projection of $\left(\xi_{\eta}^{ \pm}\right)_{3}$ on $I^{ \pm}$. We have $\zeta_{\eta}^{ \pm} \in W^{1, \infty}\left(S ; \mathbb{R}^{3}\right) \subset H^{1 / 2}\left(S ; \mathbb{R}^{3}\right)$ and $\left|\zeta_{\eta}^{ \pm}-\zeta^{ \pm}\right|_{L^{p} m\left(s, \mathbb{R}^{3}\right)} \leq C \eta$, so that, the field $u_{s}$ of $V_{s}$ defined by

$$
u_{s}(x)=\left\{\begin{array}{l}
u\left(x \mp \varepsilon e_{3}\right) \text { in } \Omega_{\varepsilon}^{ \pm} \\
R_{\varepsilon}\left(\zeta_{\eta}^{+}-\zeta_{\eta}^{-}\right)(x) \text { in } B_{\varepsilon}
\end{array}\right.
$$

does, because of $\left(H_{5}\right)$, satisfy

- $\int_{\Omega_{\varepsilon}} W\left(e\left(u_{s}\right)\right) d x=\int_{\Omega \backslash S} W(e(u)) d x$

- $\lim \sup \int_{B_{\varepsilon}} W_{\mu_{S}, \mu_{D}}\left(e\left(u_{s}\right)\right) d x \leq \int_{S} \bar{W}_{\bar{\mu}_{\mathbb{S}}, \bar{\mu}_{D}}\left(\zeta^{+}-\zeta^{-}\right) d \hat{x}+O(\eta)$,

- $\lim \sup \int_{S_{\varepsilon}^{ \pm}}^{ \pm} h^{ \pm}\left(\left[u_{s}\right]_{\varepsilon}^{ \pm}\right) d \hat{x} \leq \int_{S} h^{ \pm}\left( \pm\left(u^{ \pm}-\zeta^{ \pm}\right)\right) d \hat{x}+O(\eta)$

by arguing as in [19]. 
6.3.4. Proof of Proposition 6.5 and Theorem 6.1. First, we may proceed as in Proposition 3.5 by taking due account of Propositions 6.2 and 6.3, and then arguments similar to those involved in the proof of Theorem 3.1 work.

\section{References}

1. Licht C, Michaille G. A modelling of elastic adhesive bonded joints. Advances in Mathematical Sciences and Applications 1997; 7(2):711-740.

2. Aitmoussa A. Modélisation et étude des singularités de contraintes d'un joint collé très mince, Ph.D. Thesis, University Montpellier 2, France, 1989.

3. Acerbi E, Buttazzo G. Limit problems for plates surrounded by soft material. Archive for Rational Mechanics 1986; 92:355-370.

4. Geymonat G, Krasucki F, Lenci S. Mathematical analysis of a bounded joint with a soft thin adhesive. Mathematics and Mechanics of Solids 1999 ; 4:201-22.

5. Licht C, Michaille G, Pagano S. A model of elastic adhesive bonded joints through oscillation-concentration measures. Journal de Mathématiques Pures et Appliquées (9) 2007; 87(4):343-365.

6. Bayada G, Chambat M, Lhalouani K. Asymtotic analysis of a thin layer device with Tresca's contact law in elasticity. Mathematical Methods in the Applied Sciences 1999; 22:811-836.

7. Zaittouni F. Modélisation théorique et numérique d'interfaces, Ph.D. thesis, University Montpellier 2, France, 2000.

8. Attouch H, Murat F. Homogenization of fissured elastic materials. Publication AVAMAC 85-03, Université de Perpignan, 1985.

9. Sanchez Palencia E. Nonhomogeneous media and vibration theory, Vol. 127. Springer: Berlin, 1980. Lecture notes in Physics.

10. da Silva LFM, Ochsner A, Adams RD. Handbook of Adhesion Technology. Springer-Verlag, 2011.

11. Godet M. Third bodies in Tribology. Wear 1990; 136(1):29-45.

12. Attouch H, Buttazzo G, Michaille G. Variational analysis in Sobolev and BV spaces: application to PDEs and optimization. MPS-SIAM Book Series on Optimization, 2005.

13. Rabier P, Martins JA, Oden JT, Campos L. Existence and local uniqueness of solutions to contact problems in elasticity with non linear friction laws. International Journal of Engineering Science 1986; 24:1755-1768.

14. Raous M. Friction and Instabilities, Martins JAC, Raous M (eds). Springer-Verlag, 2002. (Lectures from the Advanced Summer School on Friction and Instabilities held in Udine, July 3-7, 2000).

15. Tartar L. An Introduction to Sobolev Spaces and Interpolation Spaces, Lecture Notes of the Unione of Mathematica Italiana, vol. 3. Springer, 2007.

16. Dacorogna B. Direct methods in the calculus of variations. In Applied Mathematical Sciences, Vol. 78. Springer-Verlag: Berlin, 1989.

17. Ball J, Marsden JE. Quasi convexity at the boundary, positivity of the second variation and elastic stability. Archive for Rational Mechanics and Analysis 1984; 86:251-277.

18. Licht C. Comportement asymtotique d'une bande dissipative mince de faible rigidité. Comptes Rendus de l'Académie des Sciences Paris, t. 317 , Série I 1993; 317(4):429-433.

19. Licht C, Léger A, Orankitjaroen S, Ould Khaoua A. Dynamics of elastic bodies connected by a thin soft viscoelastic layer. Journal de Mathématiques Pures et Appliquées 2013; 99:685-703.

20. Licht C, Orankitjaroen S. Dynamics of elastic bodies connected by a thin soft inelastic layer. Comptes Rendus Mecanique 2013; 341(3):323-332. 